\title{
Small Spans in Scaled Dimension
}

\author{
John M. Hitchcock* \\ Department of Computer Science \\ University of Wyoming \\ jhitchco@cs.uwyo.edu
}

\begin{abstract}
Juedes and Lutz (1995) proved a small span theorem for polynomial-time many-one reductions in exponential time. This result says that for language $A$ decidable in exponential time, either the class of languages reducible to $A$ (the lower span) or the class of problems to which $A$ can be reduced (the upper span) is small in the sense of resource-bounded measure and, in particular, that the degree of $A$ is small. Small span theorems have been proven for increasingly stronger polynomial-time reductions, and a small span theorem for polynomial-time Turing reductions would imply BPP $\neq$ EXP. In contrast to the progress in resource-bounded measure, Ambos-Spies, Merkle, Reimann, and Stephan (2001) showed that there is no small span theorem for the resource-bounded dimension of Lutz (2003), even for polynomial-time many-one reductions.

Resource-bounded scaled dimension, recently introduced by Hitchcock, Lutz, and Mayordomo (2004), provides rescalings of resource-bounded dimension. We use scaled dimension to further understand the contrast between measure and dimension regarding polynomial-time spans and degrees. We strengthen prior results by showing that the small span theorem holds for polynomial-time many-one reductions in the $-3^{\text {rd }}$-order scaled dimension, but fails to hold in the $-2^{\text {nd }}$-order scaled dimension. Our results also hold in exponential space.

As an application, we show that determining the $-2^{\text {nd }}$ or $-1^{\text {st }}$-order scaled dimension in ESPACE of the many-one complete languages for $\mathrm{E}$ would yield a proof of $\mathrm{P}=\mathrm{BPP}$ or $\mathrm{P} \neq \mathrm{PSPACE}$. On the other hand, it is shown unconditionally that the complete languages for $\mathrm{E}$ have $-3^{\text {rd }}$-order scaled dimension 0 in ESPACE and $-2^{\text {nd }}-$ and $-1^{\text {st }}$-order scaled dimension 1 in $\mathrm{E}$.
\end{abstract}

\section{Introduction}

Resource-bounded measure [16] defines the relative size of classes of decision problems and has been used very successfully to study polynomial-time reductions within exponential-time complexity classes. Measure-theoretic arguments were the first to show that for all $\alpha<1$, every $\leq_{n^{\alpha}-t^{\mathrm{p}}}^{\mathrm{p}}$-hard language for exponential time is exponentially dense [19]. The first plausible hypothesis on NP to separate the $\leq_{\mathrm{m}}^{\mathrm{p}}$ and $\leq_{\mathrm{T}}^{\mathrm{p}}$ reducibilities within NP came from resource-bounded measure [20].

The degrees and spans of languages under polynomial-time reductions have also been studied by several researchers using resource-bounded measure. For a reducibility $\leq_{r}^{\mathrm{p}}$ and any $A \subseteq\{0,1\}^{*}$, the $\leq_{r}^{\mathrm{p}}$-lower span of $A$ is the class $\mathrm{P}_{r}(A)$ of all languages that are $\leq_{r}^{\mathrm{p}}$-reducible to $A$, the $\leq_{r}^{\mathrm{p}}$-upper span of $A$ is the class $\mathrm{P}_{r}^{-1}(A)$ of all languages to which $A$ is $\leq_{r}^{\mathrm{p}}$-reducible, and the $\leq_{r}^{\mathrm{p}}$-degree of

\footnotetext{
*This research was supported in part by National Science Foundation Grant 9988483.
} 
$A$ is the class $\operatorname{deg}_{r}^{\mathrm{p}}(A)=\mathrm{P}_{r}(A) \cap \mathrm{P}_{r}^{-1}(A)$. Juedes and Lutz [12] proved the following small span theorem for $\leq \mathrm{p}_{\mathrm{m}}^{\mathrm{p}}$ reductions in both $\mathrm{E}$ and in EXP. Here the notation $\mu(\mathcal{C} \mid \mathcal{D})$ denotes the measure of $\mathcal{C}$ within $\mathcal{D}$, where $\mathcal{D}$ is a suitable complexity class. If $\mu(\mathcal{C} \mid \mathcal{D})=0$, then intuitively $\mathcal{C} \cap \mathcal{D}$ is a negligible subset of $\mathcal{D}$.

Theorem 1.1. (Juedes and Lutz [12]) Let $\mathcal{D} \in\{\mathrm{E}, \mathrm{EXP}\}$. For every $A \in \mathcal{D}$,

$$
\mu\left(\mathrm{P}_{\mathrm{m}}(A) \mid \mathcal{D}\right)=0
$$

or

$$
\mu\left(\mathrm{P}_{\mathrm{m}}^{-1}(A) \mid \mathcal{D}\right)=0
$$

In particular, $\mu\left(\operatorname{deg}_{\mathrm{m}}^{\mathrm{p}}(A) \mid \mathcal{D}\right)=0$.

That is, at least one of the upper or lower spans of $A$ is small within $\mathcal{D}$. Using a result of Bennett and Gill [4], Juedes and Lutz [12] noted that strengthening Theorem 1.1 from $\leq_{\mathrm{m}}^{\mathrm{p}}$ reductions to $\leq_{\mathrm{T}}^{\mathrm{p}}$ reductions would achieve the separation $\mathrm{BPP} \neq \mathrm{EXP}$. Pursuing this program, small span theorems for reductions of progressively increasing strength between $\leq_{\mathrm{m}}^{\mathrm{p}}$ and $\leq_{\mathrm{T}}^{\mathrm{p}}$ have been obtained by Lindner [14], Ambos-Spies, Neis, and Terwijn [3], and Buhrman and van Melkebeek [6].

Resource-bounded dimension was introduced by Lutz [18] as an effectivization of Hausdorff dimension [9] to investigate the fractal structure of complexity classes. Just like resource-bounded measure, resource-bounded dimension is defined within suitable complexity classes $\mathcal{D}$. For any complexity class $\mathcal{C}$, the dimension of $\mathcal{C}$ within $\mathcal{D}$ is a real number in $[0,1]$ and is denoted by $\operatorname{dim}(\mathcal{C} \mid \mathcal{D})$. If $\operatorname{dim}(\mathcal{C} \mid \mathcal{D})<1$, then $\mu(\mathcal{C} \mid \mathcal{D})=0$, but the converse may fail. This means that resource-bounded dimension is capable of quantitatively distinguishing among the measure 0 sets. With regard to the measure 0 sets in Theorem 1.1, Ambos-Spies, Merkle, Reimann, and Stephan [2] proved the following.

Theorem 1.2. (Ambos-Spies, Merkle, Reimann, and Stephan [2]) For every $A \in \mathrm{E}$,

$$
\operatorname{dim}\left(\operatorname{deg}_{\mathrm{m}}^{\mathrm{p}}(A) \mid \mathrm{E}\right)=\operatorname{dim}\left(\mathrm{P}_{\mathrm{m}}(A) \mid \mathrm{E}\right) .
$$

In particular, as $\operatorname{dim}(\mathrm{E} \mid \mathrm{E})=1$, the $\leq_{\mathrm{m}}^{\mathrm{p}}$-complete degree for $\mathrm{E}$ has dimension 1 within $\mathrm{E}$. This implies that replacing " $\mu$ " by "dim" in Theorem 1.1 makes the statement for E no longer true. In other words, there is no analogue of the small span theorem for dimension in E. Dimension in E cannot distinguish between lower spans and degrees.

To overcome limitations of resource-bounded dimension for investigating complexity classes within ESPACE, Hitchcock, Lutz, and Mayordomo [11] introduced for each integer $i \in \mathbb{Z}$ an $i^{\text {th }}$ order scaled dimension $\operatorname{dim}^{(i)}(\cdot \mid \mathcal{D})$. For any class $\mathcal{C}$ and $i \in \mathbb{Z}$, $\operatorname{dim}^{(i)}(\mathcal{C} \mid \mathcal{D}) \in[0,1]$, and if it is less than 1 , then $\mu(\mathcal{C} \mid \mathcal{D})=0$. The quantity $\operatorname{dim}^{(i)}(\mathcal{C} \mid \mathcal{D})$ is nondecreasing in $i$, and there is at most one $i \in \mathbb{Z}$ for which $0<\operatorname{dim}^{(i)}(\mathcal{C} \mid \mathcal{D})<1$. The $0^{\text {th }}$-order dimension, $\operatorname{dim}^{(0)}(\cdot \mid \mathcal{D})$, is precisely the standard unscaled dimension, and the other orders can be more useful than it for certain complexity classes. To illustrate this, we mention some examples from circuit-size complexity. For a function $s: \mathbb{N} \rightarrow \mathbb{N}$, let $\operatorname{SIZE}(s(n))$ consist of all languages decidable by nonuniform Boolean circuit families of size at most $s(n)$. Lutz [18] showed that

$$
\operatorname{dim}\left(\operatorname{SIZE}\left(\alpha \frac{2^{n}}{n}\right) \mid \operatorname{ESPACE}\right)=\alpha
$$


for all $\alpha \in(0,1)$. Circuit size bounds of the form $2^{\alpha n}$ and $2^{n^{\alpha}}$ are typically of more interest in complexity theory, but (1.1) implies that $\operatorname{SIZE}\left(2^{\alpha n}\right)$ and $\operatorname{SIZE}\left(2^{n^{\alpha}}\right)$ have dimension 0 in ESPACE for all $\alpha \in(0,1)$. For these size bounds, the scaled dimensions are useful; in [11] it is shown that

$$
\operatorname{dim}^{(1)}\left(\operatorname{SIZE}\left(2^{\alpha n}\right) \mid \operatorname{ESPACE}\right)=\alpha
$$

and

$$
\operatorname{dim}^{(2)}\left(\operatorname{SIZE}\left(2^{n^{\alpha}}\right) \mid \operatorname{ESPACE}\right)=\alpha
$$

for any $\alpha \in(0,1)$.

This paper uses scaled dimension to investigate polynomial-time spans and degrees and further understand the contrast between Theorems 1.1 and 1.2. We show that the same dichotomy also occurs between the $-3^{\text {rd }}$ - and $-2^{\text {nd }}$-orders of scaled dimension. The main contribution of this paper is a strengthening of Theorem 1.1 to give a small span theorem for scaled dimension. (The following is a corollary of a stronger result proved in Theorem 6.3.)

Theorem 1.3. Let $\mathcal{D} \in\{\mathrm{E}, \mathrm{EXP}, \mathrm{ESPACE}, \mathrm{EXPSPACE}\}$. For every $A \in \mathcal{D}$,

$$
\operatorname{dim}^{(-3)}\left(\mathrm{P}_{\mathrm{m}}(A) \mid \mathcal{D}\right)=0
$$

or

$$
\operatorname{dim}^{(-3)}\left(\mathrm{P}_{\mathrm{m}}^{-1}(A) \mid \mathcal{D}\right)=0
$$

In particular, $\operatorname{dim}^{(-3)}\left(\operatorname{deg}_{\mathrm{m}}^{\mathrm{p}}(A) \mid \mathcal{D}\right)=0$.

In contrast, Theorem 1.2 is extended to scaled dimension at orders $i$ with $|i| \leq 2$.

Theorem 1.4. Let $\mathcal{D} \in\{\mathrm{E}, \mathrm{EXP}, \mathrm{ESPACE}, \mathrm{EXPSPACE}\}$. For every $A \in \mathcal{D}$ and $-2 \leq i \leq 2$,

$$
\operatorname{dim}^{(i)}\left(\operatorname{deg}_{\mathrm{m}}^{\mathrm{p}}(A) \mid \mathcal{D}\right)=\operatorname{dim}^{(i)}\left(\mathrm{P}_{\mathrm{m}}(A) \mid \mathcal{D}\right) .
$$

This implies that Theorem 1.3 cannot be improved to $-2^{\text {nd }}$-order scaled dimension.

As an application of these results, we consider the scaled dimension of $\mathcal{C}_{\mathrm{m}}^{\mathrm{p}}(\mathrm{E})$, the class of polynomial-time many-one complete sets for E, within ESPACE. Let $i \in\{-2,-1\}$. We extend a theorem of Lutz [15] to show that

$$
\operatorname{dim}^{(i)}\left(\mathcal{C}_{\mathrm{m}}^{\mathrm{p}}(\mathrm{E}) \mid \mathrm{ESPACE}\right)>0 \Rightarrow \mathrm{P}=\mathrm{BPP} .
$$

On the other hand, we show that

$$
\operatorname{dim}^{(i)}\left(\mathcal{C}_{\mathrm{m}}^{\mathrm{p}}(\mathrm{E}) \mid \mathrm{ESPACE}\right)<1 \Rightarrow \mathrm{P} \neq \mathrm{PSPACE}
$$

Therefore, determining the $-1^{\text {st }}$ or $-2^{\text {nd }}$-order scaled dimension of $\mathcal{C}_{\mathrm{m}}^{\mathrm{p}}(\mathrm{E})$ in ESPACE would derandomize BPP or separate $\mathrm{P}$ from PSPACE. In contrast, we also show that

$$
\operatorname{dim}^{(-3)}\left(\mathcal{C}_{\mathrm{m}}^{\mathrm{p}}(\mathrm{E}) \mid \mathrm{ESPACE}\right)=0
$$

and

$$
\operatorname{dim}^{(-2)}\left(\mathcal{C}_{\mathrm{m}}^{\mathrm{p}}(\mathrm{E}) \mid \mathrm{E}\right)=\operatorname{dim}^{(-1)}\left(\mathcal{C}_{\mathrm{m}}^{\mathrm{p}}(\mathrm{E}) \mid \mathrm{E}\right)=1
$$

hold without any hypothesis. 
This paper is organized as follows. Section 2 contains the basic preliminaries and Section 3 reviews resource-bounded scaled dimension. We develop some new tools for computing scaled dimension in Section 4. The scaled dimensions of some auxiliary classes involving polynomial reductions are calculated in Section 5. Our small span theorem for scaled dimension is proved in Section 6 . Section 7 shows that lower spans and degrees have the same dimension in orders $i$ with $-2 \leq i \leq 2$. Extensions of the results to $\leq_{1-\mathrm{tt}}^{\mathrm{p}}$-reductions are discussed in Section 8. The results on the scaled dimension of the complete sets for $\mathrm{E}$ are presented in Section 9. Section 10 concludes with a brief summary.

\section{Preliminaries}

The set of all finite binary strings is $\{0,1\}^{*}$. The empty string is denoted by $\lambda$. We use the standard enumeration of binary strings $s_{0}=\lambda, s_{1}=0, s_{2}=1, s_{3}=00, \ldots$. The length of a string $x \in\{0,1\}^{*}$ is denoted by $|x|$. We use the notation $\{0,1\}^{\leq n}=\left\{x \in\{0,1\}^{*}|| x \mid \leq n\right\}$ and $\{0,1\}^{>n}=\left\{x \in\{0,1\}^{*}|| x \mid>n\right\}$.

All languages (decision problems) in this paper are encoded as subsets of $\{0,1\}^{*}$. For a language $A \subseteq\{0,1\}^{*}$, we define $A_{\leq n}=\{x \in A|| x \mid \leq n\}$. We routinely identify $A$ with its infinite binary characteristic sequence according to the standard enumeration of binary strings. We write $A\lceil n$ for the $n$-bit prefix of the characteristic sequence of $A$, and $A[n]$ for the $n^{\text {th }}$-bit of its characteristic sequence.

Let $\leq_{r}^{\mathrm{p}}$ be a polynomial-time reducibility. For any $A \subseteq\{0,1\}^{*}$, let

$$
\mathrm{P}_{r}(A)=\left\{B \subseteq\{0,1\}^{*} \mid B \leq_{r}^{\mathrm{p}} A\right\}
$$

be the $\leq_{r}^{\mathrm{p}}$-lower span of $A$,

$$
\mathrm{P}_{r}^{-1}(A)=\left\{B \subseteq\{0,1\}^{*} \mid A \leq_{r}^{\mathrm{p}} B\right\}
$$

be the $\leq_{r}^{\mathrm{p}}$-upper span of $A$, and

$$
\operatorname{deg}_{r}^{\mathrm{p}}(A)=\mathrm{P}_{r}(A) \cap \mathrm{P}_{r}^{-1}(A)
$$

be the $\leq_{r}^{\mathrm{p}}$-degree of $A$. For any complexity class $\mathcal{D}$, the class of $\leq_{r}^{\mathrm{p}}$-hard languages for $\mathcal{D}$ is

$$
\mathcal{H}_{r}^{\mathrm{p}}(\mathcal{D})=\left\{A \subseteq\{0,1\}^{*} \mid \mathcal{D} \subseteq \mathrm{P}_{r}(A)\right\},
$$

and the class of $\leq_{r}^{\mathrm{p}}$-complete languages for $\mathcal{D}$ is

$$
\mathcal{C}_{r}^{\mathrm{p}}(\mathcal{D})=\mathcal{D} \cap \mathcal{H}_{r}^{\mathrm{p}}(\mathcal{D}) .
$$

Let resource $\in\{$ time, space $\}$ and let $t(n)$ be a resource bound. Let $l \in \mathbb{N}$. A function $f: \mathbb{N}^{l} \times$ $\{0,1\}^{*} \rightarrow[0, \infty) \cap \mathbb{Q}$ is $t(n)$-resource exactly computable if there is a Turing machine that computes $f\left(k_{1}, \ldots, k_{l}, w\right)$ using at most $t\left(k_{1}+\cdots+k_{l}+|w|\right)$ resource for all $\left(k_{1}, \ldots, k_{l}, w\right) \in \mathbb{N}^{l} \times\{0,1\}^{*}$. Let $g: \mathbb{N}^{l} \times\{0,1\}^{*} \rightarrow[0, \infty)$ be a real-valued function. An approximation of $g$ is a function $\hat{g}: \mathbb{N}^{l+1} \times\{0,1\}^{*} \rightarrow[0, \infty)$ such that

$$
|g(x)-\hat{g}(r, x)| \leq 2^{-r}
$$

for all $x \in \mathbb{N}^{l} \times\{0,1\}^{*}$ and $r \in \mathbb{N}$. We say that $g$ is $t(n)$-resource computable if there is an exactly $t(n)$-resource computable approximation $\hat{g}$ of $g$. A family of functions $\left(f_{i}: \mathbb{N}^{l} \times\{0,1\}^{*} \rightarrow[0, \infty) \mid\right.$ 
$i \in \mathbb{N}$ ) is uniformly $t(n)$-resource (exactly) computable if the function $f(i, x)=f_{i}(x)$ is $t(n)$-resource (exactly) computable.

A function $f$ is p-computable (respectively, pspace-computable) if it is $O\left(n^{k}\right)$-time (respectively, $O\left(n^{k}\right)$-space) computable for some $k \in \mathbb{N}$, and $f$ is $\mathrm{p}_{2}$-computable (respectively, $\mathrm{p}_{2}$ space-computable) if it is $O\left(2^{(\log n)^{k}}\right)$-time (respectively, $O\left(2^{(\log n)^{k}}\right)$-space) computable for some $k \in \mathbb{N}$. Throughout this paper, unless otherwise specified, $\Delta$ denotes any of the resource bounds $\mathrm{p}, \mathrm{p}_{2}$, pspace, or $\mathrm{p}_{2}$ space. The concept of an exactly $\Delta$-computable function is defined analogously.

\section{Scaled Dimension}

Hitchcock, Lutz, and Mayordomo [11] introduced resource-bounded scaled dimension. This section briefly reviews the essentials of this theory.

The principle concept is a scale, which is a function $g: H \times[0, \infty) \rightarrow \mathbb{R}$, where $H=(a, \infty)$ for some $a \in \mathbb{R} \cup\{-\infty\}$. A scale must satisfy certain properties that are given in [11] and will not be discussed here. The canonical example of a scale is the function $g_{0}: \mathbb{R} \times[0, \infty) \rightarrow \mathbb{R}$ defined by $g_{0}(m, s)=s m$. This scale is used in the standard (unscaled) dimension. Other scales of interest are obtained from $g_{0}$ by rescaling and reflection operations.

Definition. Let $g: H \times[0, \infty) \rightarrow \mathbb{R}$ be a scale.

1. The first rescaling of $g$ is the scale $g^{\#}: H^{\#} \times[0, \infty) \longrightarrow \mathbb{R}$ defined by

$$
\begin{gathered}
H^{\#}=\left\{2^{m} \mid m \in H\right\} \\
g^{\#}(m, s)=2^{g(\log m, s)} .
\end{gathered}
$$

2. The reflection of $g$ is the scale $g^{R}: H \times[0, \infty) \rightarrow \mathbb{R}$ defined by

$$
g^{R}(m, s)= \begin{cases}m+g(m, 0)-g(m, 1-s) & \text { if } 0 \leq s \leq 1 \\ g(m, s) & \text { if } s \geq 1\end{cases}
$$

A family of scales, one for each integer, is defined as follows.

Definition. 1. For each $i \in \mathbb{N}$, define $a_{i}$ by the recurrence $a_{0}=-\infty, a_{i+1}=2^{a_{i}}$.

2. For each $i \in \mathbb{Z}$, define the $i^{\text {th }}$ scale $g_{i}:\left(a_{|i|}, \infty\right) \times[0, \infty) \rightarrow \mathbb{R}$ by the following recursion.

(a) $g_{0}(m, s)=s m$

(b) For $i \geq 0, g_{i+1}=g_{i}^{\#}$.

(c) For $i<0, g_{i}=g_{-i}^{R}$. by

For clarity, we compute the first few scales. For all $s \in[0,1]$, if $m>a_{|i|}$, then $g_{i}(m, s)$ is defined

$$
\begin{aligned}
g_{3}(m, s) & =2^{2^{(\log \log m)^{s}}} \\
g_{2}(m, s) & =2^{(\log m)^{s}} \\
g_{1}(m, s) & =m^{s} \\
g_{0}(m, s) & =s m \\
g_{-1}(m, s) & =m+1-m^{1-s} \\
g_{-2}(m, s) & =m+2-2^{(\log m)^{1-s}} \\
g_{-3}(m, s) & =m+4-2^{2^{(\log \log m)^{1-s}}} .
\end{aligned}
$$


Scaled dimension is defined using functions called scaled gales. The more familiar concepts of gales [18] and martingales [16] are special cases in the following definition.

Definition. Let $i \in \mathbb{Z}$ and let $s \in[0, \infty)$.

1. An $i^{\text {th }}$-order scaled $s$-gale (briefly, an $s^{(i)}$-gale) is a function $d:\{0,1\}^{>a_{|i|}} \rightarrow[0, \infty)$ such that for all $w \in\{0,1\}^{*}$ with $|w|>a_{|i|}$,

$$
d(w)=2^{-\Delta g_{i}(|w|, s)}[d(w 0)+d(w 1)],
$$

where $\Delta g_{i}:\left(a_{|i|}, \infty\right) \times[0, \infty) \rightarrow \mathbb{R}$ is defined by

$$
\Delta g_{i}(m, s)=g_{i}(m+1, s)-g_{i}(m, s) .
$$

2. An $s$-gale is an $s^{(0)}$-gale, that is, a function $d:\{0,1\}^{*} \rightarrow[0, \infty)$ satisfying

$$
d(w)=2^{-s}[d(w 0)+d(w 1)]
$$

for all $w \in\{0,1\}^{*}$.

3. A martingale is a 1-gale, that is, a function $d:\{0,1\}^{*} \rightarrow[0, \infty)$ satisfying

$$
d(w)=\frac{d(w 0)+d(w 1)}{2}
$$

for all $w \in\{0,1\}^{*}$.

Success sets are a crucial concept for resource-bounded measure, and also for scaled dimension.

Definition. Let $d:\{0,1\}^{>a} \rightarrow[0, \infty)$, where $a \in \mathbb{Z}$.

1. We say that $d$ succeeds on a language $A \subseteq\{0,1\}^{*}$ if

$$
\limsup _{n \rightarrow \infty} d(A\lceil n)=\infty .
$$

2. The success set of $d$ is

$$
S^{\infty}[d]=\left\{A \subseteq\{0,1\}^{*} \mid d \text { succeeds on } A\right\}
$$

Resource-bounded measure is defined using success sets of martingales. Here $\Delta$ denotes any of the resource bounds $\left\{\mathrm{p}, \mathrm{p}_{2}\right.$, pspace, $\mathrm{p}_{2}$ space $\}$, and $R(\Delta)$ is the following exponential-time or -space complexity class.

$$
\begin{aligned}
& R(\mathrm{p})=\mathrm{E}=\operatorname{DTIME}\left(2^{O(n)}\right) \\
& R\left(\mathrm{p}_{2}\right)=\operatorname{EXP}=\operatorname{DTIME}\left(2^{n^{O(1)}}\right) \\
& R(\text { pspace })=\operatorname{ESPACE}=\operatorname{DSPACE}\left(2^{O(n)}\right) \\
& R\left(\mathrm{p}_{2} \text { space }\right)=\operatorname{EXPSPACE}=\operatorname{DSPACE}\left(2^{n^{O(1)}}\right)
\end{aligned}
$$

Definition. Let $\mathcal{C}$ be a class of languages.

1. We say that $\mathcal{C}$ has $\Delta$-measure 0 , and write $\mu_{\Delta}(\mathcal{C})=0$, if there is a $\Delta$-computable martingale $d$ such that $\mathcal{C} \subseteq S^{\infty}[d]$. 
2. We say that $\mathcal{C}$ has measure 0 in $R(\Delta)$, and write $\mu(\mathcal{C} \mid R(\Delta))=0$, if $\mu_{\Delta}(\mathcal{C} \cap R(\Delta))=0$.

The measure conservation theorem of Lutz [16] asserts that $\mu_{\Delta}(R(\Delta)) \neq 0$, justifying the definition of measure in $R(\Delta)$ above.

Success sets of scaled gales are used to define scaled dimension.

Definition. Let $\mathcal{C}$ be a class of languages and $i \in \mathbb{Z}$.

1. The $i^{\text {th }}$-order scaled $\Delta$-dimension of $\mathcal{C}$ is

$$
\operatorname{dim}_{\Delta}^{(i)}(\mathcal{C})=\inf \left\{\begin{array}{l|l}
s & \begin{array}{l}
\text { there exists a } \Delta \text {-computable } \\
s^{(i)} \text {-gale } d \text { for which } \mathcal{C} \subseteq S^{\infty}[d]
\end{array}
\end{array}\right\}
$$

2. The $i^{\text {th }}$-order scaled dimension of $\mathcal{C}$ within $R(\Delta)$ is

$$
\operatorname{dim}^{(i)}(\mathcal{C} \mid R(\Delta))=\operatorname{dim}_{\Delta}^{(i)}(\mathcal{C} \cap R(\Delta)) .
$$

The $0^{\text {th }}$-order dimension $\operatorname{dim}_{\Delta}^{(0)}(\cdot)$ is precisely the dimension $\operatorname{dim}_{\Delta}(\cdot)$ of Lutz [18], and the other orders are interpreted as rescalings of this concept.

The following lemma relates resource-bounded scaled dimension to resource-bounded measure.

Lemma 3.1. ([11]) For any class $\mathcal{C}$ of languages and $i \in \mathbb{Z}$,

$$
\operatorname{dim}_{\Delta}^{(i)}(\mathcal{C})<1 \Rightarrow \mu_{\Delta}(\mathcal{C})=0
$$

and

$$
\operatorname{dim}^{(i)}(\mathcal{C} \mid R(\Delta))<1 \Rightarrow \mu(\mathcal{C} \mid R(\Delta))=0 .
$$

The following is another key property of scaled dimension.

Theorem 3.2. ([11]) Let $\mathcal{C}$ be a class of languages and $i \in \mathbb{Z}$. If $\operatorname{dim}_{\Delta}^{(i+1)}(\mathcal{C})<1$, then $\operatorname{dim}_{\Delta}^{(i)}(\mathcal{C})=$ 0 .

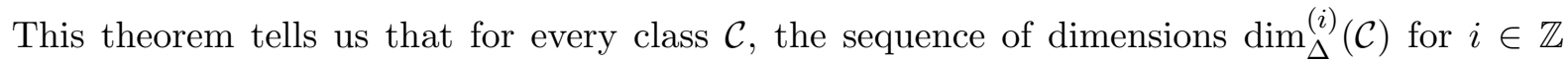
satisfies exactly one of the following three conditions.

(i) $\operatorname{dim}_{\Delta}^{(i)}(\mathcal{C})=0$ for all $i \in \mathbb{Z}$.

(ii) $\operatorname{dim}_{\Delta}^{(i)}(\mathcal{C})=1$ for all $i \in \mathbb{Z}$.

(iii) There exist $i^{*} \in \mathbb{Z}$ such that $\operatorname{dim}_{\Delta}^{(i)}(\mathcal{C})=0$ for all $i<i^{*}$ and $\operatorname{dim}_{\Delta}^{(i)}(\mathcal{C})=1$ for all $i>i^{*}$.

\section{Measures, Log-Loss, and Scaled Dimension}

This section provides some tools involving measures and the log-loss concept that are useful for working with the scaled dimensions. It was shown in [10] that log-loss unpredictability is equivalent to dimension. Here we characterize scaled dimension using the log-loss of measures. A similar approach to classical fractal dimension using measures has been used by Cutler [7] (see also [8]). 
Definition. A measure is a function $\rho:\{0,1\}^{*} \rightarrow[0, \infty)$ satisfying

$$
\rho(w)=\rho(w 0)+\rho(w 1)
$$

for all $w \in\{0,1\}^{*}$.

Measures have the following fundamental relationship with scaled gales. This extends Schnorr's "likelihood ratio" characterization of martingales [23].

Observation 4.1. Let $i \in \mathbb{Z}$ and $s \in[0, \infty)$.

1. If $\rho:\{0,1\}^{*} \rightarrow[0, \infty)$ is a measure, then the function $d_{\rho}:\{0,1\}^{>a_{|i|}} \rightarrow[0, \infty)$ defined by

$$
d_{\rho}(w)=2^{g_{i}(|w|, s)} \rho(w)
$$

for all $w \in\{0,1\}^{>a_{|i|}}$ is an $s^{(i)}$-gale.

2. If $d:\{0,1\}^{>a|i|} \rightarrow[0, \infty)$ is an $s^{(i)}$-gale, then the function $\rho_{d}:\{0,1\}^{*} \rightarrow[0, \infty)$ defined by

$$
\rho_{d}(w)=2^{-g_{i}(|w|, s)} d(w)
$$

for all $w \in\{0,1\}^{>a_{|i|}}$ and

$$
\rho_{d}(w)=\sum_{|v|=a_{|i|}+1-|w|} \rho_{d}(w v)
$$

for all $w \in\{0,1\}^{\leq a_{|i|}}$ is a measure.

The following lemma relates the scaled dimension of a class to limits involving scales and logarithms of measures.

Lemma 4.2. Let $\mathcal{C}$ be a class of languages and let $i \in \mathbb{Z}$.

1. If $s>\operatorname{dim}_{\Delta}^{(i)}(\mathcal{C})$, then there is a $\Delta$-computable measure $\rho$ such that

$$
\limsup _{n \rightarrow \infty}\left[g_{i}(n, s)+\log \rho(A\lceil n)]=\infty\right.
$$

for all $A \in \mathcal{C}$.

2. If $s<\operatorname{dim}_{\Delta}^{(i)}(\mathcal{C})$, then for any $\Delta$-computable measure $\rho$ there is an $A_{\rho} \in \mathcal{C}$ such that

$$
\lim _{n \rightarrow \infty}\left[g_{i}(n, s)+\log \rho\left(A_{\rho}\lceil n)\right]=-\infty .\right.
$$

Proof. Let $r$ be rational with $s>r>\operatorname{dim}_{\Delta}^{(i)}(\mathcal{C})$ and let $d$ be a $\Delta$-computable $r^{(i)}$-gale succeeding on $\mathcal{C}$. Then the measure $\rho_{d}$ from Observation 4.1 is also $\Delta$-computable. Let $A \in \mathcal{C}$. There are infinitely many $n \in \mathbb{N}$ such that $d\left(A\lceil n) \geq 1\right.$ since $A \in S^{\infty}[d]$. For such $n$,

$$
\begin{aligned}
g_{i}(n, s)+\log \rho_{d}(A\lceil n) & =g_{i}(n, s)-g_{i}(n, r)+\log d(A\lceil n) \\
& \geq g_{i}(n, s)-g_{i}(n, r) .
\end{aligned}
$$

Part 1 follows because $r<s$. 
For part 2 , let $\rho$ be a $\Delta$-computable measure. Let $t$ be rational with $s<t<\operatorname{dim}_{\Delta}^{(i)}(\mathcal{C})$ and obtain the $t^{(i)}$-gale $d_{\rho}$ from Observation 4.1. Then $\mathcal{C} \nsubseteq S^{\infty}\left[d_{\rho}\right]$ because $d_{\rho}$ is $\Delta$-computable, so there is an $A_{\rho} \in \mathcal{C}$ and a constant $c$ such that $d\left(A\lceil n) \leq c\right.$ for all $n>a_{|i|}$. Then

$$
\begin{aligned}
g_{i}(n, s)+\log \rho(A\lceil n) & =g_{i}(n, s)-g_{i}(n, t)+\log d_{\rho}(A\lceil n) \\
& \leq g_{i}(n, s)-g_{i}(n, t)+\log c,
\end{aligned}
$$

so the claim follows because $s<t$.

Lemma 4.2 asserts that if the $i^{\text {th }}$-order scaled dimension of a class $\mathcal{C}$ is less than $s$ then there is a measure $\rho$ such that for every $A \in \mathcal{C}$, there are prefixes $w \sqsubseteq A$ where the log-loss quantity

$$
-\log \rho(w)
$$

is arbitrarily less than $g_{i}(|w|, s)$.

It is often convenient to replace computable measures by exactly computable measures. The following lemma is proved in the same way as the Exact Computation Lemma for martingales [13].

Lemma 4.3. Let $\rho$ be a measure that is computable in $t(n)$ time (respectively, space), where $t(n) \geq$ $n$ is nondecreasing. Then there is a measure $\tilde{\rho}$ that is exactly computable in $n \cdot t(2 n+2)$ time (respectively, space) such that $\tilde{\rho}(w) \geq \rho(w)$ for all $w \in\{0,1\}^{*}$.

The measures that are exactly computable within a fixed time or space bound are uniformly exactly computable with slightly more time or space.

Lemma 4.4. For any nondecreasing time constructible function $t(n) \geq n$ the family of exactly $t(n)$-time computable measures is uniformly exactly computable in $O\left(n^{2} t(n) \log t(n)\right)$ time. The family of exactly $t(n)$-space computable measures is uniformly exactly computable in $O(t(n))$ space.

Proof. There is a uniform enumeration $\left(M_{i} \mid i \in \mathbb{N}\right)$ of all $t(n)$-time clocked Turing machines such that for all $i \in \mathbb{N}, M_{i}(w)$ can be computed in $i \cdot t(|w|) \log t(|w|)$ time for all $w \in\{0,1\}^{*}$. Define $\rho_{i}:\{0,1\}^{*} \rightarrow[0, \infty)$ inductively by $\rho_{i}(\lambda)=M_{i}(\lambda)$ and

$$
\begin{gathered}
\rho_{i}(w 0)= \begin{cases}M_{i}(w 0) & \text { if } M_{i}(w 0) \leq \rho_{i}(w) \\
\rho_{i}(w) & \text { otherwise }\end{cases} \\
\rho_{i}(w 1)=\rho_{i}(w)-\rho_{i}(w 0)
\end{gathered}
$$

for all $w \in\{0,1\}^{*}$. Then each $\rho_{i}$ is a measure. Also, if $\nu$ is a measure that is exactly computed by $M_{i}$ in $t(n)$ time, then $\rho_{i}(w)=\nu(w)$ for all $w$. We can compute $\rho_{i}(w)$ by using $|w|$ computations of $M_{i}$ on strings of length at most $|w|$, so the function $\rho: \mathbb{N} \times\{0,1\}^{*} \rightarrow[0, \infty)$ defined by $\rho(i, w)=\rho_{i}(w)$ is computable in $O\left(n^{2} t(n) \log t(n)\right)$ time. The argument for space is similar.

Uniformly exactly computable families of measures can be combined into a single measure in an efficient manner.

Lemma 4.5. Let $\left(\rho_{k} \mid k \in \mathbb{N}\right)$ be a uniformly exactly $\Delta$-computable family of measures. There is a $\Delta$-computable measure $\rho^{*}$ such that for any $k$, there is a constant $c_{k}$ such that

$$
\log \rho^{*}(w) \geq \log \rho_{k}(w)-c_{k}
$$

for all $w \in\{0,1\}^{*}$. 
Proof. Define

$$
\rho^{*}(w)=\sum_{k=0}^{\infty} \frac{\rho_{k}(w)}{2^{k} \rho_{k}(\lambda)} .
$$

Then $\rho$ is a measure by linearity. Also, $\rho^{*}$ is $\Delta$-computable by the approximation function $\hat{\rho}^{*}$ : $\mathbb{N} \times\{0,1\}^{*} \rightarrow[0, \infty)$ defined by

$$
\hat{\rho}^{*}(r, w)=\sum_{k=0}^{r} \frac{\rho_{k}(w)}{2^{k} \rho_{k}(\lambda)}
$$

since

$$
\begin{aligned}
\left|\rho^{*}(w)-\hat{\rho}^{*}(r, w)\right| & =\sum_{k=r+1}^{\infty} \frac{\rho_{k}(w)}{2^{k} \rho_{k}(\lambda)} \\
& \leq \sum_{k=r+1}^{\infty} \frac{\rho_{k}(\lambda)}{2^{k} \rho_{k}(\lambda)} \\
& =2^{-r} .
\end{aligned}
$$

Let $k \in \mathbb{N}$. For any $w \in\{0,1\}^{*}$,

$$
\begin{aligned}
\log \rho^{*}(w) & \geq \log \frac{\rho_{k}(w)}{2^{k} \rho_{k}(\lambda)} \\
& =\log \rho_{k}(w)-k-\rho_{k}(\lambda),
\end{aligned}
$$

so the lemma holds with $c_{k}=k+\rho_{k}(\lambda)$.

We now combine the preceding lemmas to obtain a tool that will be useful in calculating scaled dimensions.

Theorem 4.6. Let $\mathcal{C}$ be a class of languages, $i \in \mathbb{Z}$, and $k \in \mathbb{N}$.

1. If for each $A \in \mathcal{C}$ there is a measure $\rho_{A}$ computable in $O\left(n^{k}\right)$ time such that

$$
\left(\exists c_{A} \in \mathbb{Z}\right)\left(\exists^{\infty} n\right) g_{i}(n, s)+\log \rho_{A}\left(A\lceil n) \geq c_{A},\right.
$$

then $\operatorname{dim}_{\mathrm{p}}^{(i)}(\mathcal{C}) \leq s$.

2. If for each $A \in \mathcal{C}$ there is a measure $\rho_{A}$ computable in $O\left(2^{(\log n)^{k}}\right)$ time such that (4.1) holds, then $\operatorname{dim}_{\mathrm{P}_{2}}^{(i)}(\mathcal{C}) \leq s$.

3. If for each $A \in \mathcal{C}$ there is a measure $\rho_{A}$ computable in $O\left(n^{k}\right)$ space such that (4.1) holds, then $\operatorname{dim}_{\text {pspace }}^{(i)}(\mathcal{C}) \leq s$

4. If for each $A \in \mathcal{C}$ there is a measure $\rho_{A}$ computable in $O\left(2^{(\log n)^{k}}\right)$ space such that (4.1) holds, then $\operatorname{dim}_{\mathrm{P}_{2} \text { space }}^{(i)}(\mathcal{C}) \leq s$.

Proof. From Lemmas $4.3,4.4$, and 4.5 we obtain an exactly $\Delta$-computable measure $\rho$ such that $\log \rho(w) \geq \log \rho_{A}(w)-b_{A}$ for all $w \in\{0,1\}^{*}$ where $b_{A}$ is a constant that depends on $A$ but not on $w$. 
Let $t>s$. For any $A \in \mathcal{C}$,

$$
g_{i}(n, t)+\log \rho\left(A\lceil n) \geq g_{i}(n, t)-g_{i}(n, s)+c_{A}-b_{A}\right.
$$

for infinitely many $n$. Therefore

$$
\limsup _{n \rightarrow \infty} g_{i}(n, t)+\log \rho(A\lceil n)=\infty
$$

since $t>s$. It follows from the contrapositive of Lemma $4.2(2)$ that $\operatorname{dim}_{\Delta}(\mathcal{C}) \leq t$.

\section{Scaled Non-Bi-Immunity and Compressibility}

In this section we introduce some classes involving scales, non-bi-immunity, and compressibility by polynomial-time reductions and calculate their scaled dimensions.

A Turing machine $M$ is consistent with a language $A \subseteq\{0,1\}^{*}$ if for all $x \in\{0,1\}^{*}$,

$$
M(x) \text { halts } \Longleftrightarrow M(x)=A(x) .
$$

Let $t$ be a time bound. The fast set of $M$ with respect to $t$ is

$$
F_{M}^{t}=\left\{x \in\{0,1\}^{*} \mid \operatorname{time}_{M}(x) \leq t(|x|)\right\} .
$$

Recall that $A$ is not $\operatorname{DTIME}(t)$-bi-immune if there is a machine $M$ consistent with $A$ such that $F_{M}^{t}$ is infinite.

Definition. For any time bound $t$, let $X(t)$ be the class of all languages that are not DTIME $(t)$ bi-immune.

Let $A \subseteq\{0,1\}^{*}$ and $f:\{0,1\}^{*} \rightarrow\{0,1\}^{*}$. We say that $f$ is a many-one reduction of $A$ if there is some $B \subseteq\{0,1\}^{*}$ such that $x \in A \Longleftrightarrow f(x) \in B$. The collision set of $f$ is

$$
C_{f}=\left\{s_{i} \mid(\exists j<i) f\left(s_{i}\right)=f\left(s_{j}\right)\right\} .
$$

Recall that $A$ is compressible by $\leq_{\mathrm{m}}^{\mathrm{DTIME}(t)}$-reductions if there exists an $f \in \operatorname{DTIMEF}(t)$ that is a many-one reduction of $A$ and has $C_{f}$ infinite [12].

Definition. For any time bound $t$, let $C(t)$ be the class of all languages that are compressible by $\leq_{\mathrm{m}}^{\mathrm{DTIME}(t)}$-reductions.

The following theorem asserts that almost every language in $\mathrm{E}$ is $\operatorname{DTIME}\left(2^{c n}\right)$-bi-immune [21] and incompressible by $\leq_{\mathrm{m}}^{\mathrm{DTIME}}\left(2^{c n}\right)$-reductions [12].

Theorem 5.1. (Mayordomo [21], Juedes and Lutz [12]) For all $c \in \mathbb{N}$,

$$
\mu_{\mathrm{p}}\left(X\left(2^{c n}\right)\right)=\mu_{\mathrm{p}}\left(C\left(2^{c n}\right)\right)=0
$$

and

$$
\mu_{\mathrm{p}_{2}}\left(X\left(2^{n^{c}}\right)\right)=\mu_{\mathrm{p}_{2}}\left(C\left(2^{n^{c}}\right)\right)=0 .
$$

The next two definitions introduce scaled versions of $X(t)$ and $C(t)$. 
Definition. For any $i \in \mathbb{Z}, \alpha \in[0,1]$, and time bound $t$, let

$$
X_{\alpha}^{(i)}(t)=\left\{A \subseteq\{0,1\}^{*} \begin{array}{l}
(\exists M) M \text { is consistent with } A \text { and } \\
\left(\exists^{\infty} n\right) \#\left(1, F_{M}^{t}\lceil n) \geq n-g_{i}(n, \alpha)\right.
\end{array}\right\}
$$

That is, $X_{\alpha}^{(i)}(t)$ consists of the languages that are not DTIME $(t)$-bi-immune in a particular strong way: for infinitely many $n$, all but $g_{i}(n, \alpha)$ of the first $n$ strings can be decided in less than $t$ time by a consistent Turing machine.

Definition. For any $i \in \mathbb{Z}, \alpha \in[0,1]$, and time bound $t$, let

$$
C_{\alpha}^{(i)}(t)=\left\{\begin{array}{l|l}
A \in\{0,1\}^{*} & \begin{array}{l}
(\exists f \in \operatorname{DTIMEF}(t)) f \text { is a many-one reduction of } A \\
\text { and }\left(\exists^{\infty} n\right) \#\left(1, C_{f}\lceil n) \geq n-g_{i}(n, \alpha)\right.
\end{array}
\end{array}\right\} .
$$

In other words, $C_{\alpha}^{(i)}(t)$ is the class of languages compressible by $\leq_{\mathrm{m}}^{\text {DTIME }(t)}$-reductions where for infinitely many $n$, all but $g_{i}(n, \alpha)$ of the first $n$ strings have downward collisions under some reduction.

For $\alpha<1, X_{\alpha}^{(i)}\left(2^{n}\right) \subseteq X\left(2^{n}\right)$ and $C_{\alpha}^{(i)}\left(2^{n}\right) \subseteq C\left(2^{n}\right)$, so Theorem 5.1 implies that $X_{\alpha}^{(i)}\left(2^{n}\right)$ and $C_{\alpha}^{(i)}\left(2^{n}\right)$ have measure 0 . We now refine this by calculating their scaled dimensions.

Theorem 5.2. For all $i \in \mathbb{Z}, c \geq 1$, and $\alpha \in[0,1]$,

$$
\operatorname{dim}_{\mathrm{p}}^{(i)}\left(X_{\alpha}^{(i)}\left(2^{c n}\right)\right)=\operatorname{dim}_{\mathrm{p}}^{(i)}\left(C_{\alpha}^{(i)}\left(2^{c n}\right)\right)=\alpha
$$

and

$$
\operatorname{dim}_{\mathrm{p}_{2}}^{(i)}\left(X_{\alpha}^{(i)}\left(2^{n^{c}}\right)\right)=\operatorname{dim}_{\mathrm{P}_{2}}^{(i)}\left(C_{\alpha}^{(i)}\left(2^{n^{c}}\right)\right)=\alpha .
$$

Proof. We focus on the p-dimension portion of the theorem; the argument for $\mathrm{p}_{2}$-dimension is identical. Let $\alpha \in(0,1)$ and let $s, t>0$ be arbitrary rationals with $s<\alpha<t$. It suffices to show that

$$
s \leq \operatorname{dim}_{\mathrm{p}}^{(i)}\left(X_{\alpha}^{(i)}\left(2^{c n}\right)\right) \leq \operatorname{dim}_{\mathrm{p}}^{(i)}\left(C_{\alpha}^{(i)}\left(2^{c n}\right)\right) \leq t .
$$

The inequality $\operatorname{dim}_{\mathrm{p}}^{(i)}\left(X_{\alpha}^{(i)}\left(2^{c n}\right)\right) \leq \operatorname{dim}_{\mathrm{p}}^{(i)}\left(C_{\alpha}^{(i)}\left(2^{c n}\right)\right)$ holds because of the inclusion $X_{\alpha}^{(i)}\left(2^{c n}\right) \subseteq$ $C_{\alpha}^{(i)}\left(2^{c n}\right)$.

For the lower bound, let $\rho$ be any p-computable measure; assume without loss of generality that $\rho(\lambda) \leq 1$. We define a language $A$ inductively by lengths. Let $s<s^{\prime \prime}<s^{\prime}<\alpha$ with $s^{\prime}$ rational. The first $\left\lceil g_{i}\left(2^{n}, s^{\prime}\right)\right\rceil$ bits of $A_{=n}$ are set by diagonalization to minimize $\rho$. The remaining $2^{n}-\left\lceil g_{i}\left(2^{n}, s^{\prime}\right)\right\rceil$ bits are identically 0 . More formally, if $x$ is the characteristic string of $A_{\leq n-1}$, we choose $v \in\{0,1\}^{\left\lceil g_{i}\left(2^{n}, s^{\prime}\right)\right\rceil}$ so that $\rho(x v)$ is minimized, and let $A_{=n}$ have characteristic string

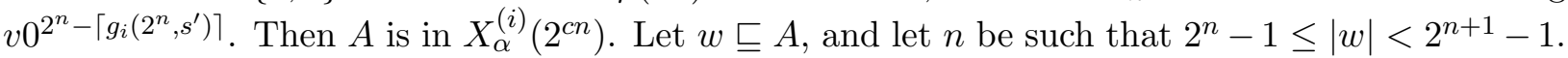
Then

$$
\log \rho(w) \leq\left(\sum_{j=0}^{n-1}-g_{i}\left(2^{j}, s^{\prime}\right)\right)-\min \left\{|w|-\left(2^{n}-1\right), g_{i}\left(2^{n}, s^{\prime}\right)\right\},
$$

which is at most $-g_{i}\left(|w|, s^{\prime \prime}\right)$ if $|w|$ is sufficiently large by Lemma 5.3 below. Then

$$
\log \rho(w)+g_{i}(|w|, s) \leq-g_{i}\left(|w|, s^{\prime \prime}\right)+g_{i}(|w|, s),
$$


so

$$
\lim _{n \rightarrow \infty} \log \rho\left(A\lceil n)+g_{i}(n, s)=-\infty\right.
$$

since $s^{\prime \prime}>s$. Since $\rho$ is an arbitrary p-computable measure, the contrapositive of Lemma 4.2(1) implies that $\operatorname{dim}_{\mathrm{p}}^{(i)}\left(X_{\alpha}^{(i)}\left(2^{c n}\right)\right) \geq s$.

Now we prove the upper bound. Let $A \in C_{\alpha}^{(i)}\left(2^{c n}\right)$ by a function $f \in \operatorname{DTIMEF}\left(2^{c n}\right)$. Define a measure $\rho$ inductively by $\rho(\lambda)=1$ and for all $w \in\{0,1\}^{*}, b \in\{0,1\}$,

1. If $f\left(s_{i}\right) \neq f\left(s_{|w|}\right)$ for all $i<|w|$, then

$$
\rho(w b)=\frac{\rho(w)}{2} .
$$

2. Otherwise, let $i=\min \left\{i<|w| \mid f\left(s_{i}\right)=f\left(s_{|w|}\right)\right\}$ and define

$$
\rho(w b)= \begin{cases}\rho(w) & \text { if } b=w[i] \\ 0 & \text { if } b \neq w[i] .\end{cases}
$$

Then for all $w \sqsubseteq A$,

$$
\begin{aligned}
\log \rho(w) & =-\#\left(0, C_{f} \uparrow|w|\right) \\
& =\#\left(1, C_{f} \uparrow|w|\right)-|w| .
\end{aligned}
$$

Whenever $\#\left(1, C_{f}\lceil n) \geq n-g_{i}(n, \alpha)\right.$, we have

$$
\begin{aligned}
g_{i}(n, t)+\log \rho(A\lceil n) & =g_{i}(n, t)+\#\left(1, C_{f}\lceil n)-n\right. \\
& \geq g_{i}(n, t)-g_{i}(n, \alpha) .
\end{aligned}
$$

This happens infinitely often, so

$$
\limsup _{n \rightarrow \infty} g_{i}(n, t)+\log \rho(A\lceil n)=\infty
$$

because $t>\alpha$. Also, $\rho$ is computable in $O\left(|w| \cdot 2^{c \log |w|}\right)=O\left(|w|^{c+1}\right)$ time. Such a $\rho$ can be defined for each $A \in C_{\alpha}^{(i)}\left(2^{c n}\right)$, so $\operatorname{dim}_{\mathrm{p}}^{(i)}\left(C_{\alpha}^{(i)}\left(2^{c n}\right)\right) \leq t$ follows by Theorem 4.6.

Lemma 5.3. Let $i \in \mathbb{Z}, 0<r<r^{\prime}<1$. Then for all sufficiently large $n$ and $k$ with $2^{n}-1 \leq k<$ $2^{n+1}-1$,

$$
\sum_{j=0}^{n-1} g_{i}\left(2^{j}, r^{\prime}\right)+\min \left\{k-\left(2^{n}-1\right), g_{i}\left(2^{n}, r^{\prime}\right)\right\} \geq g_{i}(k, r) .
$$

Proof. If $i=0$, then the left-hand side is

$$
r^{\prime}\left(2^{n}-1\right)+\min \left\{k-\left(2^{n}-1\right), r^{\prime} 2^{n}\right\} \geq r^{\prime} k=g_{0}\left(k, r^{\prime}\right)>g_{0}(k, r) .
$$

If $i=1$, then the left-hand side is

$$
\sum_{j=0}^{n-1} 2^{j r^{\prime}}+\min \left\{k-\left(2^{n}-1\right), 2^{n r^{\prime}}\right\}>2^{(n-1) r^{\prime}}>2^{(n+1) r}>k^{r}=g_{1}(k, r)
$$


when $n$ is large enough. The argument for $i>1$ is similar.

If $i=-1$, then the left-hand side is

$$
\begin{aligned}
& 2^{n}-1-\sum_{j=0}^{n-1} 2^{j\left(1-r^{\prime}\right)}+n+\min \left\{k-\left(2^{n}-1\right), 2^{n}-2^{n\left(1-r^{\prime}\right)}+1\right\} \\
& \geq k-(n+1) 2^{n\left(1-r^{\prime}\right)} \\
& \geq k-2^{n(1-r)}+1 \\
& =g_{-1}(k, r)
\end{aligned}
$$

if $n$ is sufficiently large. The argument for $i<-1$ is similar.

\section{Small Span Theorem}

In this section we establish our small span theorem for scaled dimension. We begin with a simple, but important, lemma about the scales.

Lemma 6.1. For all $k \geq 1$ and $s, t \in(0,1), g_{3}\left(2^{n^{k}}, s\right)=o\left(g_{2}\left(2^{n}, t\right)\right)$.

Proof. We have

$$
g_{3}\left(2^{n^{k}}, s\right)=2^{2^{\left(\log \log 2^{n^{k}}\right)^{s}}}=2^{2^{(k \log n)^{s}}}
$$

and

$$
g_{2}\left(2^{n}, t\right)=2^{\left(\log 2^{n}\right)^{t}}=2^{n^{t}}=2^{2^{t \log n}} .
$$

The lemma holds since $(k \log n)^{s}=o(t \log n)$.

Juedes and Lutz [12] proved that the upper spans of incompressible languages are small. Specifically, for any language $A \in \operatorname{EXP}$ that is incompressible by $\leq_{\mathrm{m}}^{\mathrm{p}}$-reductions, they showed that $\mu_{\mathrm{P}_{2}}\left(\mathrm{P}_{\mathrm{m}}^{-1}(A)\right)=0$, and if additionally $A \in \mathrm{E}$, then $\mu_{\mathrm{p}}\left(\mathrm{P}_{\mathrm{m}}^{-1}(A)\right)=0$. The following theorem is a scaled dimension analogue of this. For any $i \in \mathbb{Z}$, let

$$
C_{\alpha}^{(i)}(\text { poly })=\bigcup_{c \in \mathbb{N}} C_{\alpha}^{(i)}\left(n^{c}+c\right) .
$$

Theorem 6.2. Let $\alpha \in(0,1)$.

1. Let $\Delta \in\{\mathrm{p}$, pspace $\}$. For any $B \in R(\Delta)-C_{\alpha}^{(1)}($ poly $), \operatorname{dim}_{\Delta}^{(-3)}\left(\mathrm{P}_{\mathrm{m}}^{-1}(B)\right)=0$.

2. Let $\Delta \in\left\{\mathrm{p}_{2}, \mathrm{p}_{2}\right.$ space $\}$. For any $B \in R(\Delta)-C_{\alpha}^{(2)}($ poly $), \operatorname{dim}_{\Delta}^{(-3)}\left(\mathrm{P}_{\mathrm{m}}^{-1}(B)\right)=0$.

Proof. We first give the proof for $\Delta=\mathrm{p}$. Let $B \in \mathrm{E}-C_{\alpha}^{(1)}$ (poly) and let $M$ be a Turing machine that decides $B$ in $O\left(2^{c n}\right)$ time. Assume $B \leq_{\mathrm{m}}^{\mathrm{p}} C$ via $f$ where $f$ is computable in $n^{k}$ time almost everywhere. Then for all sufficiently large $n$,

$$
f\left(\{0,1\}^{\leq n}\right) \subseteq\{0,1\} \leq n^{k}
$$

and

$$
\left|f\left(\{0,1\}^{\leq n}\right)\right| \geq g_{1}\left(2^{n+1}-1, \alpha\right) \geq g_{1}\left(2^{n}, \alpha\right),
$$


with the latter holding because $B \notin C_{\alpha}^{(1)}$ (poly).

Let $r \in \mathbb{N}$ such that $\frac{1}{r}<\alpha$. Define $d: \mathbb{N} \rightarrow \mathbb{N}$ by $d(n)=\lfloor n / r\rfloor$. For each $n \in \mathbb{N}$ we define a measure $\rho_{n}:\{0,1\}^{*} \rightarrow[0,1]$ by

$$
\rho_{n}(\lambda)=2^{-n}
$$

and for all $w \in\{0,1\}^{*}$ and $b \in\{0,1\}$,

1. If $|w|<2^{d(n)}$ or $\left[\left(\forall i<2^{n+1}-1\right) f\left(s_{i}\right) \neq s_{|w|}\right]$, then

$$
\rho_{n}(w b)=\frac{\rho_{n}(w)}{2} .
$$

2. Otherwise, let $i=\min \left\{i<2^{n+1}-1 \mid f\left(s_{i}\right)=s_{|w|}\right\}$ and define

$$
\rho_{n}(w b)= \begin{cases}\rho_{n}(w) & \text { if } b=B[i] \\ 0 & \text { if } b \neq B[i]\end{cases}
$$

If $|w|<2^{d(n)}$, then $\rho_{n}(w)$ is computable in $O(|w|)$ time. If $|w| \geq 2^{d(n)}$, we can compute $\rho_{n}(w)$ by using $2^{n+1}-1=O\left(|w|^{n / d(n)}\right)=O\left(|w|^{r}\right)$ computations of $M$ and $f$ on strings with length at most $n=O(\log |w|)$. Therefore $\rho_{n}(w)$ is computable in $O\left(|w|^{r}\left(2^{c \log |w|}+(\log |w|)^{k}\right)\right)=O\left(|w|^{r+c}\right)$ time for all $w \in\{0,1\}^{*}$.

Let $w_{n}=C \nmid 2^{n^{k}+1}-1$ be the characteristic string of $C_{\leq n^{k}}$. Then letting

$$
m(n)=\left|\left\{j<\left|w_{n}\right| \mid\left(\forall i<2^{n+1}-1\right) f\left(s_{i}\right) \neq s_{j}\right\}\right|,
$$

we have

$$
\rho_{n}\left(w_{n}\right) \geq \rho_{n}(\lambda) 2^{-2^{d(n)}-m(n)}=2^{-2^{d(n)}-m(n)-n} .
$$

By (6.1) and (6.2), we have

$$
m(n) \leq 2^{n^{k}+1}-1-g_{1}\left(2^{n}, \alpha\right)
$$

if $n$ is sufficiently large. In this case,

$$
\log \rho_{n}\left(w_{n}\right) \geq g_{1}\left(2^{n}, \alpha\right)-2^{d(n)}-2^{n^{k}+1}-n .
$$

The function $\rho:\{0,1\}^{*} \rightarrow[0, \infty)$ defined by

$$
\rho(w)=\sum_{n=0}^{\infty} \rho_{n}(w)
$$

for all $w$ is a measure by linearity. Notice that $\rho(w)$ can be approximated to a precision of $2^{-l}$ in $O\left(|w|^{r+c} l\right)$ time by adding the first $l+1$ terms of the sum.

Using (6.3), for all sufficiently large $n$, we have

$$
\begin{aligned}
g_{-3}\left(\left|w_{n}\right|, s\right)+\log \rho_{n}\left(w_{n}\right) & =2^{n^{k}+1}+4-g_{3}\left(2^{n^{k}+1}-1,1-s\right)+\log \rho_{n}\left(w_{n}\right) \\
& \geq g_{1}\left(2^{n}, \alpha\right)-g_{3}\left(2^{n^{k}+1}-1,1-s\right)-2^{d(n)}-n .
\end{aligned}
$$


By Lemma $6.1, g_{3}\left(2^{n^{k}+1}-1,1-s\right)=o\left(g_{1}\left(2^{n}, \alpha\right)\right)$. Also, $2^{d(n)}=2^{\lfloor n / r\rfloor}$ is little- $o$ of $g_{1}\left(2^{n}, \alpha\right)=2^{\alpha n}$ because $\alpha>1 / r$. Using these facts, it follows that

$$
\limsup _{n \rightarrow \infty} g_{-3}(n, s)+\log \rho_{n}(C\lceil n)=\infty .
$$

Appealing to Theorem 4.6, we establish $\operatorname{dim}_{\mathrm{p}}^{(-3)}\left(\mathrm{P}_{\mathrm{m}}^{-1}(B)\right) \leq s$. As $s>0$ is arbitrary, the $\Delta=\mathrm{p}$ part of the theorem holds. The argument is identical for $\Delta=$ pspace.

The proof for $\Delta \in\left\{\mathrm{p}_{2}, \mathrm{p}_{2}\right.$ space $\}$ is very similar, so we only sketch the differences for $\Delta=$ $\mathrm{p}_{2}$. Let $B \in \operatorname{EXP}-C_{\alpha}^{(2)}\left(2^{n}\right)$ and let $M$ be a Turing machine that decides $B$ in $O\left(2^{n^{c}}\right)$ time. Assume $B \leq_{\mathrm{m}}^{\mathrm{p}} C$ via $f$. The measures $\rho_{n}$ and $\rho$ are defined in the same way, except we use a different function $d(n)$. For this, we let $r>1 / \alpha$ and define $d(n)=\left\lfloor n^{\epsilon}\right\rfloor$ where $\epsilon=1 / r$. Then, if $|w| \geq 2^{d(n)}$, as before we can compute $\rho_{n}(w)$ by using $2^{n+1}-1$ computations of $M$ and $f$ on strings with length at most $n=O(\log |w|)$. Since $2^{n}=2^{\left(\log 2^{n^{\epsilon}}\right)^{r}}=O\left(2^{(\log |w|)^{r}}\right)$, we can compute $\rho_{n}(w)$ in $O\left(2^{(\log |w|)^{r}} \cdot 2^{(\log |w|)^{c}}\right)=O\left(2^{(\log |w|)^{\max (r, c)}}\right)$ time. Instead of (6.3), we arrive at $\log \rho_{n}\left(w_{n}\right) \geq g_{2}\left(2^{n}, \alpha\right)-2^{d(n)}-2^{n^{k}+1}-n$. The proof is completed in the same way using the fact that $2^{d(n)}=o\left(g_{2}\left(2^{n}, \alpha\right)\right)$ because $\epsilon<\alpha$.

We are now ready to prove our main theorem.

\section{Theorem 6.3.}

1. Let $\Delta \in\{\mathrm{p}$, pspace $\}$. For every $A \in R(\Delta)$,

$$
\operatorname{dim}^{(1)}\left(\mathrm{P}_{\mathrm{m}}(A) \mid R(\Delta)\right)=0
$$

or

$$
\operatorname{dim}^{(-3)}\left(\mathrm{P}_{\mathrm{m}}^{-1}(A) \mid R(\Delta)\right)=\operatorname{dim}_{\Delta}^{(-3)}\left(\mathrm{P}_{\mathrm{m}}^{-1}(A)\right)=0 .
$$

2. Let $\Delta \in\left\{\mathrm{p}_{2}, \mathrm{p}_{2}\right.$ space $\}$. For every $A \in R(\Delta)$,

$$
\operatorname{dim}^{(2)}\left(\mathrm{P}_{\mathrm{m}}(A) \mid R(\Delta)\right)=0
$$

or

$$
\operatorname{dim}^{(-3)}\left(\mathrm{P}_{\mathrm{m}}^{-1}(A) \mid R(\Delta)\right)=\operatorname{dim}_{\Delta}^{(-3)}\left(\mathrm{P}_{\mathrm{m}}^{-1}(A)\right)=0 .
$$

Proof. Let $\Delta \in\{\mathrm{p}$, pspace $\}$ and let $A \in R(\Delta)$. As in the proof of the small span theorem in [12], we consider two cases.

(I.) Suppose that

$$
\mathrm{P}_{\mathrm{m}}(A) \cap R(\Delta) \subseteq \bigcap_{\alpha \in(0,1)} C_{\alpha}^{(1)}\left(2^{n}\right) .
$$

Then $\operatorname{dim}_{\Delta}^{(1)}\left(\mathrm{P}_{\mathrm{m}}(A) \cap R(\Delta)\right) \leq \operatorname{dim}_{\mathrm{p}}^{(1)}\left(C_{\alpha}^{(1)}\left(2^{n}\right)\right) \leq \alpha$ by Theorem 5.2 for all $\alpha \in(0,1)$, so $\operatorname{dim}^{(1)}\left(\mathrm{P}_{\mathrm{m}}(A) \mid R(\Delta)\right)=\operatorname{dim}_{\Delta}^{(1)}\left(\mathrm{P}_{\mathrm{m}}(A) \cap R(\Delta)\right)=0$.

(II.) Otherwise, there is an $\alpha \in(0,1)$ such that

$$
\mathrm{P}_{\mathrm{m}}(A) \cap R(\Delta) \not C_{\alpha}^{(1)}\left(2^{n}\right) .
$$

Let $B \in \mathrm{P}_{\mathrm{m}}(A) \cap R(\Delta)-C_{\alpha}^{(1)}\left(2^{n}\right)$. Then by Theorem $6.2, \operatorname{dim}_{\Delta}^{(-3)}\left(\mathrm{P}_{\mathrm{m}}^{-1}(B)\right)=0$. Since $\mathrm{P}_{\mathrm{m}}^{-1}(A) \subseteq \mathrm{P}_{\mathrm{m}}^{-1}(B)$, we have $\operatorname{dim}_{\Delta}^{(-3)}\left(\mathrm{P}_{\mathrm{m}}^{-1}(A)\right)=0$. 
Part 2 is proved in the same way.

Theorem 6.3 implies that there is a small span theorem for $-3^{\text {rd }}$-order scaled dimension, but it is stronger than the following.

Corollary 6.4. For every $A \in R(\Delta)$,

$$
\operatorname{dim}^{(-3)}\left(\mathrm{P}_{\mathrm{m}}(A) \mid R(\Delta)\right)=0
$$

or

$$
\operatorname{dim}^{(-3)}\left(\mathrm{P}_{\mathrm{m}}^{-1}(A) \mid R(\Delta)\right)=\operatorname{dim}_{\Delta}^{(-3)}\left(\mathrm{P}_{\mathrm{m}}^{-1}(A)\right)=0 .
$$

Proof. This follows immediately from Theorem 6.3 using Theorem 3.2.

The small span theorem of Juedes and Lutz [12] is also a corollary.

Corollary 6.5. (Juedes and Lutz [12]) Let $\Delta \in\left\{\mathrm{p}, \mathrm{p}_{2}\right\}$. For every $A \in R(\Delta)$,

$$
\mu\left(\mathrm{P}_{\mathrm{m}}(A) \mid R(\Delta)\right)=0
$$

or

$$
\mu\left(\mathrm{P}_{\mathrm{m}}^{-1}(A) \mid R(\Delta)\right)=\mu_{\Delta}\left(\mathrm{P}_{\mathrm{m}}^{-1}(A)\right)=0 .
$$

Proof. This follows immediately from Theorem 6.3 and Lemma 3.1.

We also have the following regarding the scaled dimensions of the hard languages for EXP and NP.

Corollary 6.6. 1. $\operatorname{dim}_{\mathrm{p}}^{(-3)}\left(\mathcal{H}_{\mathrm{m}}^{\mathrm{p}}(\mathrm{EXP})\right)=\operatorname{dim}_{\mathrm{p}_{2}}^{(-3)}\left(\mathcal{H}_{\mathrm{m}}^{\mathrm{p}}(\mathrm{EXP})\right)=0$.

2. If $\operatorname{dim}^{(1)}(\mathrm{NP} \mid \mathrm{E})>0$, then $\operatorname{dim}_{\mathrm{p}}^{(-3)}\left(\mathcal{H}_{\mathrm{m}}^{\mathrm{p}}(\mathrm{NP})\right)=0$.

3. If $\operatorname{dim}^{(2)}(\mathrm{NP} \mid \mathrm{EXP})>0$, then $\operatorname{dim}_{\mathrm{P}_{2}}^{(-3)}\left(\mathcal{H}_{\mathrm{m}}^{\mathrm{p}}(\mathrm{NP})\right)=0$.

Proof. Let $H \in \mathcal{C}_{\mathrm{m}}^{\mathrm{p}}(\mathrm{E})$. Then also $H \in \mathcal{C}_{\mathrm{m}}^{\mathrm{p}}(\mathrm{EXP})$, so $\mathrm{P}_{\mathrm{m}}^{-1}(H)=\mathcal{H}_{\mathrm{m}}^{\mathrm{p}}(\mathrm{EXP})$. Since $\operatorname{dim}\left(\mathrm{P}_{\mathrm{m}}(H) \mid\right.$ $\mathrm{E})=\operatorname{dim}_{\mathrm{p}}(\mathrm{E})=1$, Theorem 6.3 tells us that $\operatorname{dim}_{\mathrm{p}}\left(\mathcal{H}_{\mathrm{m}}^{\mathrm{p}}(\mathrm{EXP})\right)=\operatorname{dim}_{\mathrm{p}}\left(\mathrm{P}_{\mathrm{m}}^{-1}(H)\right)=0$.

Parts 2 and 3 follow from Theorem 6.3 using any NP-complete language $A$.

Juedes and Lutz [12] concluded from their small span theorem that every $\leq{ }_{\mathrm{m}}^{\mathrm{p}}$-degree has measure 0 in E and in EXP. From Theorem 6.3 we similarly derive a stronger version of this fact: every $\leq{ }_{\mathrm{m}}^{\mathrm{p}}$-degree actually has $-3^{\mathrm{rd}}$-order dimension 0 .

Corollary 6.7. For every $A \subseteq\{0,1\}^{*}$,

$$
\operatorname{dim}^{(-3)}\left(\operatorname{deg}_{\mathrm{m}}^{\mathrm{p}}(A) \mid R(\Delta)\right)=0 .
$$

Proof. If $\operatorname{deg}_{\mathrm{m}}^{\mathrm{p}}(A)$ is disjoint from $R(\Delta)$, then $\operatorname{dim}^{(-3)}\left(\operatorname{deg}_{\mathrm{m}}^{\mathrm{p}}(A) \mid R(\Delta)\right)=\operatorname{dim}_{\mathrm{p}}^{(-3)}(\emptyset)=0$, so assume that there is some $B \in \operatorname{deg}_{\mathrm{m}}^{\mathrm{p}}(A) \cap R(\Delta)$. Because $\operatorname{deg}_{\mathrm{m}}^{\mathrm{p}}(A)=\operatorname{deg}_{\mathrm{m}}^{\mathrm{p}}(B)=\mathrm{P}_{\mathrm{m}}(B) \cap \mathrm{P}_{\mathrm{m}}^{-1}(B)$, we have

$$
\operatorname{dim}^{(-3)}\left(\operatorname{deg}_{\mathrm{m}}^{\mathrm{p}}(A) \mid R(\Delta)\right) \leq \operatorname{dim}^{(-3)}\left(\mathrm{P}_{\mathrm{m}}(B) \mid R(\Delta)\right)
$$

and

$$
\operatorname{dim}^{(-3)}\left(\operatorname{deg}_{\mathrm{m}}^{\mathrm{p}}(A) \mid R(\Delta)\right) \leq \operatorname{dim}^{(-3)}\left(\mathrm{P}_{\mathrm{m}}^{-1}(B) \mid R(\Delta)\right) .
$$

By Corollary 6.4, we have either $\operatorname{dim}^{(-3)}\left(\mathrm{P}_{\mathrm{m}}(B) \mid R(\Delta)\right)=0$ or $\operatorname{dim}^{(-3)}\left(\mathrm{P}_{\mathrm{m}}^{-1}(B) \mid R(\Delta)\right)=0$. Therefore $\operatorname{dim}^{(-3)}\left(\operatorname{deg}_{\mathrm{m}}^{\mathrm{p}}(A) \mid R(\Delta)\right)=0$. 

$R(\Delta)$

The $\leq_{\mathrm{m}}^{\mathrm{p}}$-complete languages for any complexity class have $-3^{\text {rd }}$-order dimension 0 in every

Corollary 6.8. For any class $\mathcal{D}$ of languages, $\operatorname{dim}^{(-3)}\left(\mathcal{C}_{\mathrm{m}}^{\mathrm{p}}(\mathcal{D}) \mid R(\Delta)\right)=0$.

Proof. If $\mathcal{C}_{\mathrm{m}}^{\mathrm{p}}(\mathcal{D})=\emptyset$, this is trivial. Assume $\mathcal{C}_{\mathrm{m}}^{\mathrm{p}}(\mathcal{D}) \neq \emptyset$ and let $A \in \mathcal{C}_{\mathrm{m}}^{\mathrm{p}}(\mathcal{D})$. Then $\mathcal{C}_{\mathrm{m}}^{\mathrm{p}}(\mathcal{D}) \subseteq$ $\operatorname{deg}_{\mathrm{m}}^{\mathrm{p}}(A)$, so this follows from Corollary 6.7.

\section{Lower Spans vs. Degrees in Orders -2 Through 2}

We now present some results that stand in contrast to the small span theorem of the previous section. We begin by extending the work of Ambos-Spies, Merkle, Remainn, and Stephan [2] to show that lower spans and degrees have the same scaled dimension in orders $i$ with $|i| \leq 2$.

Theorem 7.1. For any $A \in R(\Delta)$ and $-2 \leq i \leq 2$,

$$
\operatorname{dim}^{(i)}\left(\operatorname{deg}_{\mathrm{m}}^{\mathrm{p}}(A) \mid R(\Delta)\right)=\operatorname{dim}^{(i)}\left(\mathrm{P}_{\mathrm{m}}(A) \mid R(\Delta)\right)
$$

and

$$
\operatorname{dim}_{\Delta}^{(i)}\left(\operatorname{deg}_{\mathrm{m}}^{\mathrm{p}}(A)\right)=\operatorname{dim}_{\Delta}^{(i)}\left(\mathrm{P}_{\mathrm{m}}(A)\right) .
$$

Proof. We write the proof for dimension in $R(\mathrm{p})=\mathrm{E}$; the rest of theorem is proved in the same manner. The proof is based on [2].

Let $A \in \mathrm{E}$ be decidable in $O\left(2^{c n}\right)$ time. By monotonicity, $\operatorname{dim}^{(i)}\left(\operatorname{deg}_{\mathrm{m}}^{\mathrm{p}}(A) \mid \mathrm{E}\right) \leq \operatorname{dim}^{(i)}\left(\mathrm{P}_{\mathrm{m}}(A) \mid\right.$ $\mathrm{E})$. For the other inequality, let $t>s>\operatorname{dim}^{(i)}\left(\operatorname{deg}_{\mathrm{m}}^{\mathrm{p}}(A) \mid \mathrm{E}\right)$. By Lemmas 4.2 and 4.3 , for some $l \in \mathbb{N}$ there is an exactly $n^{l}$-time computable measure $\rho$ satisfying

$$
\limsup _{m \rightarrow \infty} g_{i}(m, s)+\log \rho(C\lceil m)=\infty
$$

for all $C \in \operatorname{deg}_{\mathrm{m}}^{\mathrm{p}}(A) \cap \mathrm{E}$.

Letting $k \geq 1$ be a natural number to be specified later, we define a padding function $f$ : $\{0,1\}^{*} \rightarrow\{0,1\}^{*}$ by

$$
f(x)=0^{|x|^{k}-|x|} x
$$

for all $x$. Let $R=f\left(\{0,1\}^{*}\right)$ be the range of $f$.

Let $B \in \mathrm{P}_{\mathrm{m}}(A)$. We define another language $B^{\prime}$ as

$$
B^{\prime}=(B-R) \cup f(A) .
$$

Then $B^{\prime} \in \operatorname{deg}_{\mathrm{m}}^{\mathrm{p}}(A)$. Intuitively, $B^{\prime}$ is a language that is very similar to $B$ but has $A$ encoded sparsely in it. Define a function $\tau:\{0,1\}^{*} \rightarrow\{0,1\}^{*}$ inductively by $\tau(\lambda)=1$ and

$$
\tau(w b)= \begin{cases}\tau(w) b & \text { if } s_{|w|} \notin R \\ \tau(w) 1 & \text { if } s_{|w|} \in R \cap B^{\prime} \\ \tau(w) 0 & \text { if } s_{|w|} \in R-B^{\prime}\end{cases}
$$

for all $w \in\{0,1\}^{*}$ and $b \in\{0,1\}$. Notice that

$$
\tau(B \nmid n)=B^{\prime}\lceil n
$$


for all $n$.

Define a measure $\gamma$ by $\gamma(\lambda)=1$ and

$$
\gamma(w b)= \begin{cases}\frac{\gamma(w)}{2} & \text { if } s_{|w|} \in R \\ \frac{\rho(\tau(w) b)}{\rho(\tau(w))} \gamma(w) & \text { if } s_{|w|} \notin R\end{cases}
$$

for all $w \in\{0,1\}^{*}$ and $b \in\{0,1\}$. Intuitively, $\gamma$ is designed to have performance on $B$ that is similar to $\rho$ 's performance on $B^{\prime}$. This is done by mimicking the conditional probabilities of $\rho$ for strings that are not in $R$. Note that $\gamma(w)$ can be exactly computed in $O\left(|w| \cdot\left(|w|^{l}+2^{c \log |w|}\right)=\right.$ $O\left(|w|^{\max (l, c)+1}\right)$ time.

Let $n \in N$ and let $2^{(n-1)^{k}+1} \leq m \leq 2^{n^{k}+1}-1$. Then

$$
\begin{aligned}
\log \gamma(B\lceil m) & =\sum_{1 \leq i \leq m} \log \frac{\gamma(B\lceil i)}{\gamma(B\lceil i-1)} \\
& =\sum_{\substack{1 \leq i \leq m \\
s_{i} \notin R}} \log \frac{\rho(\tau(B \nmid i-1) B[i])}{\rho(\tau(B \nmid i-1))}+\sum_{\substack{1 \leq i \leq m \\
s_{i} \in R}} \log \frac{1}{2} \\
& =\sum_{\substack{1 \leq i \leq m \\
s_{i} \notin R}} \log \frac{\rho\left(B^{\prime} \uparrow i\right)}{\rho\left(B^{\prime} \uparrow i-1\right)}-\left|\left\{1 \leq i \leq m \mid s_{i} \in R\right\}\right| \\
& \geq \sum_{1 \leq i \leq m} \log \frac{\rho\left(B^{\prime} \uparrow i\right)}{\rho\left(B^{\prime}\lceil i-1)\right.}-\left|\left\{1 \leq i \leq 2^{n^{k}+1}-1 \mid s_{i} \in R\right\}\right| \\
& =\log \rho\left(B^{\prime}\lceil m)-\sum_{i=0}^{n} 2^{n}\right. \\
& =\log \rho\left(B^{\prime}\lceil m)-2^{n+1}+1 .\right.
\end{aligned}
$$

Now assume that $g_{i}(m, s)+\log \rho\left(B^{\prime}\lceil m) \geq 1\right.$. Then we have $g_{i}(m, t)+\log \gamma(B\lceil m) \geq 1$ if

$$
2^{n+1}+g_{i}(m, s)<g_{i}(m, t) .
$$

To establish

$$
\limsup _{n \rightarrow \infty} g_{i}(m, t)+\log \gamma(B\lceil m) \geq 1,
$$

it now suffices to show we can choose $k$ so that (7.2) holds for all sufficiently large $m$. For each $-2 \leq i \leq 2$, we now give an appropriate choice of $k$ that yields this.

- $\mathbf{i}=\mathbf{2}$ : Let $k>1 / t$. Then $g_{2}(m, t) \geq g_{2}\left(2^{(n-1)^{k}}, t\right)=2^{(n-1)^{k t}}$, so $2^{n+1}=o\left(g_{2}(m, t)\right)$ because $k t>1$. Also, $g_{2}(m, s)=o\left(g_{2}(m, t)\right)$ since $s<t$, so $(7.2)$ holds when $m$ is sufficiently large.

- $\mathbf{i}=\mathbf{1}$ : Let $k=2$. Then $g_{1}(m, t) \geq g_{1}\left(2^{(n-1)^{2}}, t\right)=2^{t(n-1)^{2}}$, so $2^{n+1}=o\left(g_{1}(m, t)\right)$. Also, $g_{1}(m, s)=o\left(g_{1}(m, t)\right)$, so (7.2) holds for sufficiently large $m$.

- $\mathbf{i}=\mathbf{0}$ : Let $k=2$. Then $g_{0}(m, t) \geq g_{0}\left(2^{(n-1)^{2}}, t\right)=t 2^{(n-1)^{2}}$, so $2^{n+1}=o\left(g_{0}(m, t)\right)$. Also, $g_{0}(m, s)=o\left(g_{0}(m, t)\right)$, so (7.2) holds for sufficiently large $m$.

- $\mathbf{i}=-\mathbf{1}$ : We have $g_{-1}(m, t)=m+1-g_{1}(m, 1-t)$, so $(7.2)$ is true if $2^{n+1}+g_{1}(m, 1-t)<$ $g_{1}(m, 1-s)$. Taking $k=2$, this follows from the argument for $i=1$ above since $1-s>1-t$. 
- $\mathbf{i}=-\mathbf{2}$ : Just as in the $i=-1$ case, $(7.2)$ is true if $2^{n+1}+g_{2}(m, 1-t)<g_{2}(m, 1-s)$. Taking $k>1 /(1-s)$, this follows from the argument for $i=2$ above since $1-s>1-t$.

For each $B \in \mathrm{P}_{\mathrm{m}}(A)$, we have given a $O\left(n^{\max (l, c)}\right)$-time computable measure $\gamma$ such that $(7.3)$ holds. By Theorem 4.6, $\operatorname{dim}^{(i)}\left(\mathrm{P}_{\mathrm{m}}(A) \mid \mathrm{E}\right) \leq t$. As $t>\operatorname{dim}^{(i)}\left(\operatorname{deg}_{\mathrm{m}}^{\mathrm{p}}(A) \mid \mathrm{E}\right)$ is arbitrary, this establishes $\operatorname{dim}^{(i)}\left(\mathrm{P}_{\mathrm{m}}(A) \mid \mathrm{E}\right) \leq \operatorname{dim}^{(i)}\left(\operatorname{deg}_{\mathrm{m}}^{\mathrm{p}}(A) \mid \mathrm{E}\right)$.

Theorem 7.1 for (unscaled) dimension was proved in [2] for $\Delta=\mathrm{p}$.

Corollary 7.2. (Ambos-Spies, Merkle, Reimann, and Stephan [2]) For any $A \in \mathrm{E}$,

$$
\operatorname{dim}\left(\operatorname{deg}_{\mathrm{m}}^{\mathrm{p}}(A) \mid \mathrm{E}\right)=\operatorname{dim}\left(\mathrm{P}_{\mathrm{m}}(A) \mid \mathrm{E}\right)
$$

and

$$
\operatorname{dim}_{\mathrm{p}}\left(\operatorname{deg}_{\mathrm{m}}^{\mathrm{p}}(A)\right)=\operatorname{dim}_{\mathrm{p}}\left(\mathrm{P}_{\mathrm{m}}(A)\right) .
$$

Theorem 7.1 implies that Theorem 6.3 cannot be improved in one respect. For any $i, j \in \mathbb{Z}$, let $\operatorname{SST}[i, j]$ be the following statement.

$\mathrm{SST}[i, j]:$ For every $A \in \mathrm{E}, \operatorname{dim}^{(i)}\left(\mathrm{P}_{\mathrm{m}}(A) \mid \mathrm{E}\right)=0$ or $\operatorname{dim}^{(j)}\left(\mathrm{P}_{\mathrm{m}}^{-1}(A) \mid \mathrm{E}\right)=0$.

Let $H \in \mathcal{C}_{\mathrm{m}}^{\mathrm{p}}(\mathrm{E})$. Then

$$
\operatorname{dim}^{(i)}\left(\mathrm{P}_{\mathrm{m}}(H) \mid \mathrm{E}\right)=\operatorname{dim}^{(i)}(\mathrm{E} \mid \mathrm{E})=1
$$

for all $i$ and $\operatorname{dim}^{(-2)}\left(\operatorname{deg}_{\mathrm{m}}^{\mathrm{p}}(H) \mid \mathrm{E}\right)=1$ by Theorem 7.1 , which in turn implies

$$
\operatorname{dim}^{(-2)}\left(\mathrm{P}_{\mathrm{m}}^{-1}(H) \mid \mathrm{E}\right)=1 .
$$

Therefore, $\operatorname{SST}[i, j]$ is false if $j \geq-2$. Theorem 6.3 says that $\operatorname{SST}[1,-3]$ is true; now we know that the -3 in it cannot be improved to -2 .

We have the following corollary regarding the classes of complete sets for E, EXP, and NP.

Corollary 7.3. Let $-2 \leq i \leq 2$.

1. $\operatorname{dim}^{(i)}\left(\mathcal{C}_{\mathrm{m}}^{\mathrm{p}}(\mathrm{E}) \mid \mathrm{E}\right)=\operatorname{dim}^{(i)}\left(\mathcal{C}_{\mathrm{m}}^{\mathrm{p}}(\mathrm{EXP}) \mid \mathrm{EXP}\right)=1$.

2. $\operatorname{dim}^{(i)}(\mathrm{NP} \mid \mathrm{E})=\operatorname{dim}^{(i)}\left(\mathcal{C}_{\mathrm{m}}^{\mathrm{p}}(\mathrm{NP}) \mid \mathrm{E}\right)$.

3. $\operatorname{dim}^{(i)}(\mathrm{NP} \mid \mathrm{EXP})=\operatorname{dim}^{(i)}\left(\mathcal{C}_{\mathrm{m}}^{\mathrm{p}}(\mathrm{NP}) \mid \mathrm{EXP}\right)$.

Proof. Let $H \in \mathcal{C}_{\mathrm{m}}^{\mathrm{p}}(\mathrm{E})$. Then $\mathcal{C}_{\mathrm{m}}^{\mathrm{p}}(\mathrm{E})=\operatorname{deg}_{\mathrm{m}}^{\mathrm{p}}(H) \cap \mathrm{E}$, $\operatorname{sodim}^{(i)}\left(\mathcal{C}_{\mathrm{m}}^{\mathrm{p}}(\mathrm{E}) \mid \mathrm{E}\right)=\operatorname{dim}^{(i)}\left(\operatorname{deg}_{\mathrm{m}}^{\mathrm{p}}(H) \mid \mathrm{E}\right)=$ $\operatorname{dim}^{(i)}\left(\mathrm{P}_{\mathrm{m}}(H) \mid \mathrm{E}\right)=\operatorname{dim}_{\mathrm{p}}^{(i)}(\mathrm{E})=1$ by Theorem 7.1. The other statements follow similarly.

We can now observe a difference between the $-3^{\text {rd }}$ - and $-2^{\text {nd }}$-order scaled dimensions regarding complete degrees. Corollaries 6.8 and 7.3 together with Theorem 3.2 tell us that for $\mathcal{D} \in\{\mathrm{E}, \mathrm{EXP}\}$,

$$
\operatorname{dim}^{(i)}\left(\mathcal{C}_{\mathrm{m}}^{\mathrm{p}}(\mathcal{D}) \mid \mathcal{D}\right)= \begin{cases}0 & \text { if } i \leq-3 \\ 1 & \text { if } i \geq-2\end{cases}
$$

and

$$
\operatorname{dim}^{(i)}\left(\mathcal{C}_{\mathrm{m}}^{\mathrm{p}}(\mathrm{NP}) \mid \mathcal{D}\right)= \begin{cases}0 & \text { if } i \leq-3 \\ \operatorname{dim}^{(i)}(\mathrm{NP} \mid \mathcal{D}) & \text { if } i \geq-2\end{cases}
$$

In Section 9 we will discuss the scaled dimension of $\mathcal{C}_{\mathrm{m}}^{\mathrm{p}}(\mathrm{E})$ within ESPACE. The following extension of Theorem 7.1 will be useful. 
Theorem 7.4. For all $-2 \leq i \leq 2$,

$$
\operatorname{dim}^{(i)}\left(\mathcal{C}_{\mathrm{m}}^{\mathrm{p}}(\mathrm{E}) \mid \mathrm{ESPACE}\right)=\operatorname{dim}^{(i)}(\mathrm{E} \mid \mathrm{ESPACE}) .
$$

Proof. We use the construction from the proof of Theorem 7.1. Let $t>s>\operatorname{dim}^{(i)}\left(\mathcal{C}_{\mathrm{m}}^{\mathrm{p}}(\mathrm{E}) \mid\right.$ ESPACE) and take an exactly $n^{l}$-space computable measure $\rho$ satisfying (7.1) for all $C \in \mathcal{C}_{\mathrm{m}}^{\mathrm{p}}(\mathrm{E})$. Fix an $A \in \mathcal{C}_{\mathrm{m}}^{\mathrm{p}}(\mathrm{E})$. For any $B \in \mathrm{E}$, the set $B^{\prime}$ constructed from $A$ and $B$ is in $\mathcal{C}_{\mathrm{m}}^{\mathrm{p}}(\mathrm{E})$. The arguments then show $\operatorname{dim}^{(i)}(\mathrm{E} \mid \mathrm{ESPACE}) \leq t$.

\section{$8 \leq_{1-\mathrm{tt}}^{\mathrm{p}}$-Lower Spans vs. $\leq_{\mathrm{m}}^{\mathrm{p}}$-Lower Spans}

Theorem 7.1 is also true for most other polynomial-time reducibilities. (This fact was mentioned in [2] for Corollary 7.2 when it was proved.) To replace $\leq_{\mathrm{m}}^{\mathrm{p}}$ by $\leq_{r}^{\mathrm{p}}$ in the theorem, we only need to have $B^{\prime} \in \operatorname{deg}_{r}^{\mathrm{p}}(A)$ for the set $B^{\prime}$ that was constructed in the proof from $B \in \mathrm{P}_{r}(A)$. In particular, Theorem 7.1 is true for the $\leq_{1-\text { tt }}^{\mathrm{p}}$ reducibility. In this section we show that this holds because of another reason: the scaled dimensions of $\leq_{1-\mathrm{tt}}^{\mathrm{p}}$-lower spans and $\leq_{\mathrm{m}}^{\mathrm{p}}$-lower spans are always the same.

The following proposition was used to show that a set is weakly $\leq_{\mathrm{m}}^{\mathrm{p}}$-complete for exponential time if and only if it is $\leq_{1-\mathrm{tt}^{-}}^{\mathrm{p}}$-complete.

Proposition 8.1. (Ambos-Spies, Mayordomo, and Zheng [1]) Let $A \leq_{1-\mathrm{tt}}^{\mathrm{p}} B$. Then there is a language $C \in \mathrm{P}$ such that

$$
\hat{A}=(A \cap C) \cup\left(A^{c} \cap C^{c}\right) \leq_{\mathrm{m}}^{\mathrm{p}} B .
$$

The idea of the following lemma also comes from [1].

Lemma 8.2. Let $i \in \mathbb{Z}$. Let $\mathcal{C}, \hat{\mathcal{C}}$ be classes of languages such that for any $A \in \mathcal{C}$, there is some $C \in R(\Delta)$ such that $\hat{A}=(A \cap C) \cup\left(A^{c} \cap C^{c}\right) \in \hat{\mathcal{C}}$. Then $\operatorname{dim}_{\Delta}^{(i)}(\mathcal{C}) \leq \operatorname{dim}_{\Delta}^{(i)}(\hat{\mathcal{C}})$.

Proof. We prove this for $\Delta=\mathrm{p}$. The other cases are proved by identical arguments.

Let $s>\operatorname{dim}_{\mathrm{p}}^{(i)}(\hat{\mathcal{C}})$ be rational and obtain $\rho$ computable in $O\left(n^{r}\right)$ time from Lemma 4.2 such that

$$
\limsup _{n \rightarrow \infty} g_{i}(n, s)+\log \rho(\hat{A}\lceil n)=\infty
$$

for all $\hat{A} \in \hat{\mathcal{C}}$.

Let $A \in \mathcal{C}$ and let $C \in \operatorname{DTIME}\left(n^{k}\right)$ such that $\hat{A}=(A \cap C) \cup\left(A^{c} \cap C^{c}\right) \in \hat{\mathcal{C}}$. Define a function $\tau:\{0,1\}^{*} \rightarrow\{0,1\}^{*}$ by

$$
\tau(w)[j]= \begin{cases}w[j] & \text { if } s_{j} \in C \\ 1-w[j] & \text { if } s_{j} \notin C\end{cases}
$$

for each $0 \leq j<|w|$. Define another measure $\rho^{\prime}$ by

$$
\rho^{\prime}(w)=\rho(\tau(w)) .
$$

Then for all $n$,

$$
\rho^{\prime}(A\lceil n)=\rho(\tau(A\lceil n))=\rho(\hat{A}\lceil n) .
$$

Therefore

$$
\limsup _{n \rightarrow \infty} g_{i}(n, s)+\log \rho^{\prime}(A\lceil n)=\infty
$$


because of (8.1). As $\rho^{\prime}$ is computable in time $O\left(|w| \cdot(\log |w|)^{k}+|w|^{r}\right)$, it follows by Theorem 4.6 that $\operatorname{dim}_{\mathrm{p}}^{(i)}(\mathcal{C}) \leq s$.

We now show that the scaled dimension of a $\leq_{\mathrm{m}}^{\mathrm{p}}$-lower span is always equal to the scaled dimension of the $\leq_{1-\mathrm{tt}}^{\mathrm{p}}$-lower span.

Theorem 8.3. Let $B \subseteq\{0,1\}^{*}$ and let $i \in \mathbb{Z}$. Then

$$
\operatorname{dim}_{\Delta}^{(i)}\left(\mathrm{P}_{\mathrm{m}}(B)\right)=\operatorname{dim}_{\Delta}^{(i)}\left(\mathrm{P}_{1-\mathrm{tt}}(B)\right)
$$

and

$$
\operatorname{dim}^{(i)}\left(\mathrm{P}_{\mathrm{m}}(B) \mid R(\Delta)\right)=\operatorname{dim}^{(i)}\left(\mathrm{P}_{1-\mathrm{tt}}(B) \mid R(\Delta)\right) .
$$

Proof. By Proposition 8.1, for each $A \in \mathrm{P}_{1-\mathrm{tt}}(B)$ there is a language $C \in \mathrm{P}$ such that $\hat{A}=$ $(A \cap C) \cup\left(A^{c} \cap C^{c}\right) \in \mathrm{P}_{\mathrm{m}}(B)$. Let $\hat{\mathcal{C}}$ be the set of all such $\hat{A}$ as $A$ ranges over $\mathrm{P}_{1-\mathrm{tt}}(B)$. Then by Lemma 8.2 ,

$$
\operatorname{dim}_{\Delta}^{(i)}\left(\mathrm{P}_{1-\mathrm{tt}}(B)\right) \leq \operatorname{dim}_{\Delta}^{(i)}(\hat{\mathcal{C}}) .
$$

As $\hat{\mathcal{C}} \subseteq \mathrm{P}_{\mathrm{m}}(B) \subseteq \mathrm{P}_{1-\mathrm{tt}}(B)$, we also have

$$
\operatorname{dim}_{\Delta}^{(i)}(\hat{\mathcal{C}}) \leq \operatorname{dim}_{\Delta}^{(i)}\left(\mathrm{P}_{\mathrm{m}}(B)\right) \leq \operatorname{dim}_{\Delta}^{(i)}\left(\mathrm{P}_{1-\mathrm{tt}}(B)\right)
$$

so the first equality holds. The proof for dimension in $R(\Delta)$ is analogous.

We can now give a stronger version of Theorem 7.1.

Corollary 8.4. For any $A \in R(\Delta)$ and $-2 \leq i \leq 2$,

$$
\begin{aligned}
\operatorname{dim}^{(i)}\left(\mathrm{P}_{\mathrm{m}}(A) \mid R(\Delta)\right) & =\operatorname{dim}^{(i)}\left(\operatorname{deg}_{\mathrm{m}}^{\mathrm{p}}(A) \mid R(\Delta)\right) \\
\operatorname{dim}^{(i)}\left(\mathrm{P}_{1-\mathrm{tt}}(A) \mid R(\Delta)\right) & =\operatorname{dim}^{(i)}\left(\operatorname{deg}_{1-\mathrm{tt}}^{\mathrm{p}}(A) \mid R(\Delta)\right),
\end{aligned}
$$

and similarly with $\operatorname{dim}^{(i)}(\cdot \mid R(\Delta))$ replaced by $\operatorname{dim}_{\Delta}^{(i)}(\cdot)$.

Proof. From Theorems 7.1 and 8.3 we have

$$
\operatorname{dim}^{(i)}\left(\operatorname{deg}_{\mathrm{m}}^{\mathrm{p}}(A) \mid R(\Delta)\right)=\operatorname{dim}^{(i)}\left(\mathrm{P}_{\mathrm{m}}(A) \mid R(\Delta)\right)=\operatorname{dim}^{(i)}\left(\mathrm{P}_{1-\mathrm{tt}}(A) \mid R(\Delta)\right) .
$$

By monotonicity, we have

$$
\operatorname{dim}^{(i)}\left(\operatorname{deg}_{\mathrm{m}}^{\mathrm{p}}(A) \mid R(\Delta)\right) \leq \operatorname{dim}^{(i)}\left(\operatorname{deg}_{1-\mathrm{tt}}^{\mathrm{p}}(A) \mid R(\Delta)\right) \leq \operatorname{dim}^{(i)}\left(\mathrm{P}_{1-\mathrm{tt}}(A) \mid R(\Delta)\right),
$$

so the corollary follows. The proof for $\operatorname{dim}_{\Delta}^{(i)}(\cdot)$ is analogous.

Theorem 8.3 also yields a strengthening of Theorem 6.3: the $\mathrm{P}_{\mathrm{m}}(A)$ in it can be replaced by $\mathrm{P}_{1-\mathrm{tt}}(A)$. In fact, it is also possible to replace the $\mathrm{P}_{\mathrm{m}}^{-1}(A)$ in Theorem 6.3 by $\mathrm{P}_{1-\mathrm{tt}}^{-1}(A)$ by extending Theorems 5.2 and 6.2 to deal with $\leq_{1-\mathrm{tt}}^{\mathrm{p}}$-reductions. We omit the details. 


\section{The Scaled Dimension of $\mathcal{C}_{\mathrm{m}}^{\mathrm{p}}(\mathrm{E})$ in ESPACE}

Lutz [17] proved a small span theorem for nonuniform Turing reductions in ESPACE. This implies that $\mathcal{C}_{\mathrm{m}}^{\mathrm{p}}(\mathrm{E})$ has measure 0 in ESPACE. In Corollary 6.8 we saw that $\mathcal{C}_{\mathrm{m}}^{\mathrm{p}}(\mathrm{E})$ actually has $-3^{\text {rd }}$-order scaled dimension 0 in ESPACE. In this section we show that determining the $-2^{\text {nd }}$ or $-1^{\text {st }}$-order scaled dimension of $\mathcal{C}_{\mathrm{m}}^{\mathrm{p}}(\mathrm{E})$ in ESPACE would yield a proof of $\mathrm{P}=\mathrm{BPP}$ or $\mathrm{P} \neq \mathrm{PSPACE}$.

The $\mathrm{P}=\mathrm{BPP}$ hypothesis was related to the measure of $\mathrm{E}$ in ESPACE by Lutz [15].

Theorem 9.1. (Lutz [15]) If $\mu(\mathrm{E} \mid \mathrm{ESPACE}) \neq 0$, then $\mathrm{P}=\mathrm{BPP}$.

We will extend this result to scaled dimension. We now recall the tools Lutz used to prove it.

Nisan and Wigderson [22] showed that BPP can be derandomized if there is a decision problem in $\mathrm{E}$ that requires exponential-size circuits to be approximately solved. The hardness of a decision problem at a given length is the minimum size of a circuit that can approximately solve it. The details of the definition of this hardness are not needed in this paper; we only need to recall existing results regarding classes of languages with exponential hardness.

Definition. Let $H_{\alpha}$ be the class of all languages that have hardness at least $2^{\alpha n}$ almost everywhere in the sense of [22].

The aforementioned derandomization of BPP can be stated as follows.

Theorem 9.2. (Nisan and Wigderson [22]) If $\mathrm{E} \cap H_{\alpha} \neq \emptyset$ for some $\alpha>0$, then $\mathrm{P}=\mathrm{BPP}$.

We will also need space-bounded Kolmogorov complexity.

Definition. Given a machine $M$, a space bound $s: \mathbb{N} \rightarrow \mathbb{N}$, a language $L \subseteq\{0,1\}^{*}$, and a natural number $n$, the s-space-bounded Kolmogorov complexity of $L_{=n}$ with respect to $M$ is

$$
\operatorname{KS}_{M}^{S}\left(L_{=n}\right)=\min \left\{|\pi| \mid M(\pi, n)=\chi_{L=n} \text { in } \leq s\left(2^{n}\right) \text { space }\right\},
$$

i.e., the length of the shortest program $\pi$ such that $M$, on input $(\pi, n)$, outputs the characteristic string of $L_{=n}$ and halts without using more than $s\left(2^{n}\right)$ workspace.

Well-known simulation techniques show that there exists a machine $U$ which is optimal in the sense that for each machine $M$ there is a constant $c$ such that for all $s, L$, and $n$ we have

$$
\mathrm{KS}_{U}^{c s+c}\left(L_{=n}\right) \leq \mathrm{KS}_{M}^{s}\left(L_{=n}\right)+c .
$$

As usual, we fix such a universal machine and omit it from the notation.

Definition. For each space bound $s: \mathbb{N} \rightarrow \mathbb{N}$ and function $f: \mathbb{N} \rightarrow \mathbb{N}$ define the complexity class

$$
\mathrm{KS}_{\mathrm{i} . \mathrm{o} .}^{s}(f)=\left\{L \subseteq\{0,1\}^{*} \mid\left(\exists^{\infty} n\right) \mathrm{KS}^{s}\left(L_{=n}\right)<f(n)\right\} .
$$

Lutz showed that $H_{\alpha}$ has measure 1 in ESPACE (i.e., that $H_{\alpha}^{c}$ has measure 0 in ESPACE) if $\alpha<1 / 3$ by showing that languages not in $H_{\alpha}$ have low space-bounded Kolmogorov complexity.

Lemma 9.3. (Lutz [15]) There exist a polynomial $q$ and a constant $c$ such that for all $0<\alpha<$ $\beta<1$,

$$
H_{\alpha}^{c} \subseteq \mathrm{KS}_{\text {i.o. }}^{q}\left(2^{n}-c 2^{(1-2 \alpha) n}+2^{\beta n}\right) .
$$


The class on the right in Lemma 9.3 has measure 0 in ESPACE [16]. The scaled dimensions of similar space-bounded Kolmogorov complexity classes were studied in [11].

Theorem 9.4. (Hitchcock, Lutz, and Mayordomo [11]) For any $i \leq-1$, polynomial $q(n)=\Omega\left(n^{2}\right)$, and $\alpha \in[0,1]$,

$$
\operatorname{dim}^{(i)}\left(\operatorname{KS}_{\text {i.o. }}^{q}\left(g_{i}\left(2^{n}, \alpha\right)\right) \mid \operatorname{ESPACE}\right)=\alpha .
$$

Lemma 9.3 and Theorem 9.4 provide an easy upper bound on the $-1^{\text {st }}$-order scaled dimension of $H_{\alpha}^{c}$ in ESPACE.

Corollary 9.5. If $0<\alpha<1 / 3$, then

$$
\operatorname{dim}^{(-1)}\left(H_{\alpha}^{c} \mid \mathrm{ESPACE}\right) \leq 2 \alpha
$$

Proof. Let $\epsilon>0$ and $\beta \in(\alpha, 1-2 \alpha)$. Then for all sufficiently large $n$,

$$
\begin{aligned}
2^{n}-c 2^{(1-2 \alpha) n}+2^{\beta n} & <2^{n}+1-2^{(1-2 \alpha-\epsilon) n} \\
& =g_{-1}\left(2^{n}, 2 \alpha+\epsilon\right),
\end{aligned}
$$

so Lemma 9.3 implies $H_{\alpha}^{c} \subseteq \mathrm{KS}_{\text {i.o. }}^{q}\left(g_{-1}\left(2^{n}, 2 \alpha+\epsilon\right)\right)$. Therefore $\operatorname{dim}^{(-1)}\left(H_{\alpha}^{c} \mid\right.$ ESPACE $) \leq 2 \alpha+\epsilon$ by Theorem 9.4.

We can now state a stronger version of Theorem 9.1. The hypothesis has been weakened, but the conclusion remains the same.

Theorem 9.6. If $\operatorname{dim}^{(-1)}(\mathrm{E} \mid \mathrm{ESPACE})>0$, then $\mathrm{P}=\mathrm{BPP}$.

Proof. Assume the hypothesis and let $s=\min \left\{1 / 2, \operatorname{dim}^{(-1)}(\mathrm{E} \mid \mathrm{ESPACE})\right\}$. Then by Corollary 9.5, $\mathrm{E} \not \subset H_{s / 2}^{c}$, i.e., $\mathrm{E} \cap H_{s / 2} \neq \emptyset$. Therefore $\mathrm{P}=\mathrm{BPP}$ by Theorem 9.2.

We now relate the scaled dimension of $\mathcal{C}_{\mathrm{m}}^{\mathrm{p}}(\mathrm{E})$ to the $\mathrm{P} \stackrel{?}{=} \mathrm{PSPACE}$ and $\mathrm{P} \stackrel{?}{=} \mathrm{BPP}$ problems.

Theorem 9.7. For $i \in\{-2,-1\}$,

$$
\operatorname{dim}^{(i)}\left(\mathcal{C}_{\mathrm{m}}^{\mathrm{p}}(\mathrm{E}) \mid \mathrm{ESPACE}\right)<1 \Rightarrow \mathrm{P} \neq \mathrm{PSPACE}
$$

and

$$
\operatorname{dim}^{(i)}\left(\mathcal{C}_{\mathrm{m}}^{\mathrm{p}}(\mathrm{E}) \mid \mathrm{ESPACE}\right)>0 \Rightarrow \mathrm{P}=\mathrm{BPP} .
$$

Proof. From Theorem 7.4 we know that $\operatorname{dim}^{(i)}\left(\mathcal{C}_{\mathrm{m}}^{\mathrm{p}}(\mathrm{E}) \mid \mathrm{ESPACE}\right)=\operatorname{dim}^{(i)}(\mathrm{E} \mid \mathrm{ESPACE})$. Also, $\operatorname{dim}^{(i)}(\mathrm{E} \mid \mathrm{ESPACE})<1$ implies $\mathrm{E} \neq \mathrm{ESPACE}$ which implies $\mathrm{P} \neq \mathrm{PSPACE}[5]$. This proves the first implication. The second one follows from Theorem 9.6 since $\operatorname{dim}^{(i)}\left(\mathcal{C}_{\mathrm{m}}^{\mathrm{p}}(\mathrm{E}) \mid \mathrm{ESPACE}\right)>0$ implies $\operatorname{dim}^{(-1)}(\mathrm{E} \mid \mathrm{ESPACE})>0$.

In other words, establishing any nontrivial upper or lower bound on $\operatorname{dim}^{(-1)}\left(\mathcal{C}_{\mathrm{m}}^{\mathrm{p}}(\mathrm{E}) \mid \mathrm{ESPACE}\right)$ or $\operatorname{dim}^{(-2)}\left(\mathcal{C}_{\mathrm{m}}^{\mathrm{p}}(\mathrm{E}) \mid\right.$ ESPACE) would derandomize BPP or separate $\mathrm{P}$ from PSPACE. This is in contrast to the unconditional facts from Corollaries 6.7 and 7.3 that

$$
\operatorname{dim}^{(-3)}\left(\mathcal{C}_{\mathrm{m}}^{\mathrm{p}}(\mathrm{E}) \mid \mathrm{E}\right)=0
$$

and

$$
\operatorname{dim}^{(-2)}\left(\mathcal{C}_{\mathrm{m}}^{\mathrm{p}}(\mathrm{E}) \mid \mathrm{E}\right)=\operatorname{dim}^{(-1)}\left(\mathcal{C}_{\mathrm{m}}^{\mathrm{p}}(\mathrm{E}) \mid \mathrm{E}\right)=1
$$




\section{Conclusion}

Our main results, Theorems 6.3 and 7.1, use resource-bounded scaled dimension to strengthen from both ends the contrasting theorems of Juedes and Lutz [12] and Ambos-Spies, Merkle, Reimann, and Stephan [2] regarding spans under polynomial-time reductions.

1. The small span theorem for $\leq_{\mathrm{m}}^{\mathrm{p}}$-reductions [12] was strengthened from measure to $-3^{\text {rd }}$-order scaled dimension. (In fact, Theorem 6.3 is even stronger than this.)

2. The result that lower spans and degrees have the same dimension [2] was extended to all orders $-2 \leq i \leq 2$ of scaled dimension. This implies that there is no small span theorem in $-2^{\text {nd }}$-order scaled dimension.

These results suggest that the contrast between the $-2^{\text {nd }}$ - and $-3^{\text {rd }}$-orders of resource-bounded scaled dimension will be useful for studying complexity classes involving polynomial-time reductions. For example, regarding the many-one complete degree of NP, Corollaries 6.7 and 7.3 say that

$$
\operatorname{dim}^{(-3)}\left(\mathcal{C}_{\mathrm{m}}^{\mathrm{p}}(\mathrm{NP}) \mid \mathrm{E}\right)=0
$$

and

$$
\operatorname{dim}^{(-2)}\left(\mathcal{C}_{\mathrm{m}}^{\mathrm{p}}(\mathrm{NP}) \mid \mathrm{E}\right)=\operatorname{dim}^{(-2)}(\mathrm{NP} \mid \mathrm{E}) .
$$

Scaled dimension therefore provides two different types of dimension for studying NP. The NPcomplete degree provides all the dimension of NP in order -2 , but in order -3 the NP-complete degree unconditionally has dimension 0 .

Acknowledgments. I thank Jack Lutz for suggesting this line of research and Xiaoyang Gu for comments on a draft. I also thank the anonymous referees for very helpful comments and corrections.

\section{References}

[1] K. Ambos-Spies, E. Mayordomo, and X. Zheng. A comparison of weak completeness notions. In Proceedings of the Eleventh IEEE Conference on Computational Complexity, pages 171-178. IEEE Computer Society, 1996.

[2] K. Ambos-Spies, W. Merkle, J. Reimann, and F. Stephan. Hausdorff dimension in exponential time. In Proceedings of the 16th IEEE Conference on Computational Complexity, pages 210217. IEEE Computer Society, 2001.

[3] K. Ambos-Spies, H.-C. Neis, and S. A. Terwijn. Genericity and measure for exponential time. Theoretical Computer Science, 168(1):3-19, 1996.

[4] C. H. Bennett and J. Gill. Relative to a random oracle $A, \mathrm{P}^{A} \neq \mathrm{NP}^{A} \neq$ co-NP $\mathrm{NP}^{A}$ with probability 1. SIAM Journal on Computing, 10:96-113, 1981.

[5] R. V. Book. Comparing complexity classes. Journal of Computer and System Sciences, 9:213$229,1974$.

[6] H. Buhrman and D. van Melkebeek. Hard sets are hard to find. Journal of Computer and System Sciences, 59(2):327-345, 1999. 
[7] C. D. Cutler. Strong and weak duality principles for fractal dimension in Euclidean space. Mathematical Proceedings of the Cambridge Philosophical Society, 118:393-410, 1995.

[8] K. Falconer. Techniques in Fractal Geometry. John Wiley \& Sons, 1997.

[9] F. Hausdorff. Dimension und äußeres Maß. Mathematische Annalen, 79:157-179, 1919.

[10] J. M. Hitchcock. Fractal dimension and logarithmic loss unpredictability. Theoretical Computer Science, 304(1-3):431-441, 2003.

[11] J. M. Hitchcock, J. H. Lutz, and E. Mayordomo. Scaled dimension and nonuniform complexity. Journal of Computer and System Sciences, 69(2):97-122, 2004.

[12] D. W. Juedes and J. H. Lutz. The complexity and distribution of hard problems. SIAM Journal on Computing, 24(2):279-295, 1995.

[13] D. W. Juedes and J. H. Lutz. Weak completeness in E and $\mathrm{E}_{2}$. Theoretical Computer Science, 143(1):149-158, 1995.

[14] W. Lindner. On the polynomial time bounded measure of one-truth-table degrees and pselectivity. Diplomarbeit, Technische Universität Berlin, 1993.

[15] J. H. Lutz. An upward measure separation theorem. Theoretical Computer Science, 81(1):127$135,1991$.

[16] J. H. Lutz. Almost everywhere high nonuniform complexity. Journal of Computer and System Sciences, 44(2):220-258, 1992.

[17] J. H. Lutz. A small span theorem for P/Poly-Turing reductions. In Proceedings of the Tenth Annual Structure in Complexity Theory Conference, pages 324-330. IEEE Computer Society, 1995.

[18] J. H. Lutz. Dimension in complexity classes. SIAM Journal on Computing, 32(5):1236-1259, 2003.

[19] J. H. Lutz and E. Mayordomo. Measure, stochasticity, and the density of hard languages. SIAM Journal on Computing, 23(4):762-779, 1994.

[20] J. H. Lutz and E. Mayordomo. Cook versus Karp-Levin: Separating completeness notions if NP is not small. Theoretical Computer Science, 164(1-2):141-163, 1996.

[21] E. Mayordomo. Almost every set in exponential time is P-bi-immune. Theoretical Computer Science, 136(2):487-506, 1994.

[22] N. Nisan and A. Wigderson. Hardness vs randomness. Journal of Computer and System Sciences, 49:149-167, 1994.

[23] C. P. Schnorr. A survey of the theory of random sequences. In R. E. Butts and J. Hintikka, editors, Basic Problems in Methodology and Linguistics, pages 193-211. D. Reidel, 1977. 